\title{
Hotspot SF3B1 mutations induce metabolic reprogramming and vulnerability to serine deprivation
}

\author{
W. Brian Dalton, ${ }^{1}$ Eric Helmenstine, ${ }^{1}$ Noel Walsh, ${ }^{1}$ Lukasz P. Gondek, ${ }^{1}$ Dhanashree S. Kelkar, ${ }^{2}$ Abigail Read, ${ }^{3}$ Rachael Natrajan, ${ }^{3}$ \\ Eric S. Christenson, ${ }^{1}$ Barbara Roman, ${ }^{4}$ Samarjit Das, ${ }^{4,5}$ Liang Zhao, ${ }^{6}$ Robert D. Leone, ${ }^{6}$ Daniel Shinn, ${ }^{1}$ Taylor Groginski, ${ }^{1}$ \\ Anil K. Madugundu, ${ }^{2,7,8}$ Arun Patil,, ${ }^{2,7}$ Daniel J. Zabransky, ${ }^{1}$ Arielle Medford,, ${ }^{1,9}$ Justin Lee, ${ }^{1}$ Alex J. Cole, ${ }^{1}$ Marc Rosen, ${ }^{1}$ Maya Thakar, \\ Alexander Ambinder, ${ }^{1}$ Joshua Donaldson, ${ }^{1}$ Amy E. DeZern, ${ }^{1}$ Karen Cravero, ${ }^{1}$ David Chu, ${ }^{1}$ Rafael Madero-Marroquin, ${ }^{1,10}$ \\ Akhilesh Pandey, ${ }^{1,2,7,8,11}$ Paula J. Hurley, ${ }^{1,12}$ Josh Lauring, ${ }^{1,13}$ and Ben Ho Park, ${ }^{1,14,15}$ \\ 'Department of Oncology, The Sidney Kimmel Comprehensive Cancer Center, and ${ }^{2}$ McKusick-Nathans Institute of Genetic Medicine, Department of Biological Chemistry, Johns Hopkins University School \\ of Medicine, Baltimore, Maryland, USA. ${ }^{3}$ The Breast Cancer Now Toby Robins Research Centre, The Institute of Cancer Research, London, United Kingdom. ${ }^{4}$ Department of Pathology, Cardiovascular \\ Division, ${ }^{5}$ Department of Anesthesiology and Critical Care Medicine, and ${ }^{6}$ Bloomberg-Kimmel Institute for Cancer Immunotherapy, Johns Hopkins School of Medicine, Baltimore, Maryland, USA. Institute \\ of Bioinformatics, International Technology Park, Bangalore, India. ${ }^{8}$ Manipal Academy of Higher Education (MAHE), Manipal, Karnataka, India. ${ }^{9}$ Department of Medicine, Massachusetts General Hospital \\ and Harvard Medical School, Boston, Massachusetts, USA. ${ }^{0}$ Department of Medicine, Icahn School of Medicine, Mount Sinai St. Luke's Roosevelt Hospital Center, New York, New York, USA. "'Department of \\ Pathology and ${ }^{12}$ Department of Urology, Johns Hopkins University School of Medicine, Baltimore, Maryland, USA. ${ }^{13}$ Janssen Research and Development, Spring House, Pennsylvania, USA. ${ }^{14}$ Department of \\ Chemical and Biomolecular Engineering, The Whiting School of Engineering, Johns Hopkins University, Baltimore, Maryland, USA. ${ }^{15}$ Division of Hematology, Oncology, Department of Medicine, Vanderbilt \\ Ingram Cancer Center, Nashville, Tennessee, USA.
}

\begin{abstract}
Cancer-associated mutations in the spliceosome gene SF3B1 create a neomorphic protein that produces aberrant mRNA splicing in hundreds of genes, but the ensuing biologic and therapeutic consequences of this missplicing are not well understood. Here we have provided evidence that aberrant splicing by mutant SF3B1 altered the transcriptome, proteome, and metabolome of human cells, leading to missplicing-associated downregulation of metabolic genes, decreased mitochondrial respiration, and suppression of the serine synthesis pathway. We also found that mutant SF3B1 induces vulnerability to deprivation of the nonessential amino acid serine, which was mediated by missplicing-associated downregulation of the serine synthesis pathway enzyme PHGDH. This vulnerability was manifest both in vitro and in vivo, as dietary restriction of serine and glycine in mice was able to inhibit the growth of SF3B1 ${ }^{M U T}$ xenografts. These findings describe a role for SF3B1 mutations in altered energy metabolism, and they offer a new therapeutic strategy against SF3B $1^{\mathrm{MUT}}$ cancers.
\end{abstract}

\section{Introduction}

Since their identification in 2011, mutations in spliceosome genes have been found in numerous human malignancies (1). Among the most broadly implicated genes is $S F 3 B 1$, recurrently mutated in myelodysplastic syndrome (MDS), chronic lymphocytic leukemia, uveal melanoma, breast carcinoma, pancreas adenocarcinoma, lung adenocarcinoma, prostate adenocarcinoma, and many other cancers (2-8). Part of the U2snRNP complex, SF3B1 normally serves in the proper selection of $3^{\prime}$ acceptor sequences in pre-mRNA splicing reactions. In cancer, $S F 3 B 1$ mutations create a neomorphic spliceosome protein that induces cryptic 3 ' splice site selection by favoring an alternative branch point sequence $(9$,

Conflict of interest: WBD receives research support from Eli Lilly. J Lauring receives salary and stock from Janssen. BP receives royalties from Horizon Discovery LTD; is a scientific advisory board member for and has ownership interest in Loxo Oncology; is a paid consultant for Foundation Medicine Inc., Jackson Laboratories, H3 Biomedicine, Casdin Capital, Roche, Eli Lilly, and Astra Zeneca; and has research contracts with Abbvie, Foundation Medicine Inc., and Pfizer.

Copyright: ( 2019, American Society for Clinical Investigation.

Submitted: September 20, 2018; Accepted: August 1, 2019;

Published: September 30, 2019.

Reference information: J Clin Invest. 2019;129(11):4708-4723.

https://doi.org/10.1172/JCl125022.
10). The resulting aberrant splice junctions frequently lead to nonsense-mediated decay (NMD) and downregulation of mRNA from hundreds of affected genes (9). Yet, less is known about how these splicing alterations affect the downstream cellular processes and oncogenic properties of $S F 3 B 1^{M U T}$ cells.

Despite this still nascent understanding of its biology, mutant $S F 3 B 1$ has already become a target for novel therapeutic strategies, as many $S F 3 B 1^{M U T}$ cancers have few effective treatments (11). However, unlike canonical oncogene targets like BCR-ABL, SF3B1 mutations do not seem to create "oncogene addiction," as cancer cells with native $S F 3 B 1$ mutation do not require the continued presence of mutant protein for their in vitro viability $(11,12)$. Therefore, rather than directly inhibiting the mutant protein, successful therapeutic strategies may come from a synthetic lethal approach, whereby the treatment targets a process whose disruption would be tolerated by normal cells, but detrimental to those with an SF3B1 mutation. Among the most attractive processes to target in this way are those that are nonessential to normal cells, as this will minimize toxicity of the treatment and facilitate its combination with other therapies. To date, processes whose disruption is deleterious to $S F 3 B 1^{M U T}$ cells but nonessential to normal cells have not been identified.

Here we report investigations into the downstream functional consequences of $S F 3 B 1$ mutations in human cells, providing 
evidence that mutant $S F 3 B 1$ induces a specific remodeling of the proteome, cryptic splicing-induced downregulation of metabolic genes, and reprogramming of cellular metabolism. We also find that SF3B1 mutations create a heightened sensitivity to deprivation of the nonessential amino acid serine, suggesting a novel therapeutic strategy for targeting $S F 3 B 1^{M U T}$ cancers.

\section{Results}

Knockin of mutant SF3B1 alters the transcriptome of breast epithelial cells. SF3B1 is recurrently mutated in $2 \%$ of breast cancers and associates with poor prognosis in subgroups of patients, but the functional role of the mutation in this cellular context has been unexplored $(13,14)$. We therefore first aimed to characterize the isolated consequences of SF3B1 mutations in untransformed human breast epithelial cells. We used AAV-mediated gene targeting to engineer heterozygous knockins of the dominant K70OE hotspot in spontaneously immortalized but nontumorigenic MCF-10A breast epithelial cells. To control for nonspecific effects of gene targeting, we also generated knockins of a synonymous base substitution at R702 that is a single nucleotide polymorphism (SNP) in the human population (15), and targeted WT (TWT) clones, where cells experience gene targeting, but remain WT (Supplemental Figure 1A; supplemental material available online with this article; https://doi.org/10.1172/JCI125022DS1). Four mutant and 4 control clones were independently isolated, and heterozygosity of the knockins was confirmed at both the DNA and mRNA levels (Supplemental Figure 1B).

Splice junction analysis of whole transcriptome RNA-seq from 2 mutant and 2 control clones revealed $S F 3 B 1^{K 700 E}$-dependent alternative splicing. At a $P$ value less than 0.01 and FDR less than 0.05 , we identified 685 junctions whose usage was upregulated in $S F 3 B 1^{\text {K70OE }}$ cells (Supplemental Table 1). Alternative $3^{\prime}$ splice site selection (A3SS) was the most frequent splicing event (49\% of all junctions) (Figure 1A), with enrichment of cryptic acceptors 10-30 nucleotides upstream of canonical ones (Figure 1B). More than half $(56 \%)$ of the $S F 3 B 1^{K 700 E}$-enriched junctions were not annotated in transcriptome databases of normal human tissue (Figure $1 \mathrm{~A}$ ), compared with $12 \%$ of junctions overall, demonstrating enrichment of novel junctions in $S F 3 B 1^{K 700 E}$ cells. To orthogonally validate these RNA-seq data in all mutant and control MCF-10A clones, PCR was performed on candidate aberrant junctions in the metabolic genes MUT (methylmalonyl-CoA mutase) and DLST (dihydrolipoamide S-succinyltransferase) (Figure 1C). To contextualize these findings in another genetic background, we also created an $S F 3 B 1^{K 700 E}$ knockin clone from human mammary epithelial cells immortalized by telomerase (hTERT-IMECs) (Supplemental Figure 1C). These cells exhibited the same cryptic splicing events seen in our MCF-10A knockins (Supplemental Figure 2A). We then determined how whole-gene mRNA expression is affected by this aberrant splicing. At a $\log 2 \mathrm{FC}$ of \pm 0.5 and FDR less than 0.01 , we identified 301 downregulated and 231 upregulated genes in SF3B1 ${ }^{\text {MUT }}$ cells (Supplemental Tables 2 and 3). Downregulated genes were more frequently subject to aberrant splicing than upregulated ones: $13 \%$ (38/301) versus $2.6 \%$ (6/231), respectively $\left(\chi^{2} P<0.0001\right)$ (Figure 1D and Supplemental Tables 1 and 2). Mechanistically, this association was likely driven by nonsense-mediated mRNA decay (NMD), as 76\% (29/38) of downregulated and misspliced genes were affected by at least one aberrant junction predicted to be NMD-sensitive, compared with none of those upregulated and misspliced (Figure 1D and Supplemental Tables 1 and 3). Thus, mutant SF3B1 induces aberrant splicing dominated by cryptic $3^{\prime}$ site selection and NMD-associated mRNA downregulation in untransformed breast epithelial cells, extending observations from transformed cell lines (9-11).

To evaluate the disease relevance of these findings, we compared the junction usage profile in our MCF-10A model with that of $S F 3 B 1^{M U T}$ human breast tumors from TCGA, utilizing the analysis of Darman et al. (9). Of the 262 junctions upregulated in $S F 3 B 1^{\text {MUT }}$ breast tumors in their study, $43 \%$ (113/262) were among the 685 junctions increased in our $S F 3 B 1^{K 700 E}$ knockins, a highly significant overlap (Figure 1E and Supplemental Table 1). We then compared the whole-gene mRNA expression of our model with that of 2 independent transcriptome data sets of human breast tumors: TCGA and METABRIC $(16,17)$. Of the genes downregulated in SF3B1 ${ }^{\text {MUT }}$ MCF-10A cells, $32 \%, 21 \%$, and $17 \%$ were also downregulated in $S F 3 B 1^{M U T}$ tumors from METABRIC, TCGA, or both data sets (Figure 1E and Supplemental Table 4), respectively. Notably, 66\% (25/38) of genes downregulated and misspliced in MCF-10A cells were also among those downregulated in one or more of the $S F 3 B 1^{M U T}$ breast tumor data sets (Figure $1 \mathrm{E}$ and Supplemental Table 4), demonstrating the contextual robustness of missplicing-associated downregulation of mRNA. In contrast, overlap was lower between upregulated genes in SF3B1 ${ }^{\text {MUT }}$ MCF$10 \mathrm{~A}$ cells and breast tumors (Figure $1 \mathrm{E}$ and Supplemental Table 5). Taken together, these findings validate the disease relevance of our isogenic cell model and provide experimental evidence for determining which transcriptome alterations are directly caused by mutant $S F 3 B 1$ in human breast tumors.

Mutant SF3B1 reshapes the proteome of breast epithelial cells. Although transcriptional effects of SF3B1 mutation have now been observed in several contexts, less is known about how these alterations affect the proteome. We therefore performed peptide mass spectrometry on isogenic MCF-10A cells using stable isotope labeling of amino acids in culture (SILAC). This experiment generated abundance values for 5,715 proteins. Using a $\log ^{2}$ abundance ratio cutoff of \pm 0.5 , we identified nearly twice as many proteins downregulated by $S F 3 B 1^{K 700 E}$ than upregulated: 381 versus 204 (Supplemental Tables 6 and 7). Integrating these results with our transcriptome analysis revealed that downregulated proteins exhibited missplicing of corresponding mRNA more frequently than upregulated ones: $13 \%$ (51/396) versus 3.0\% (6/203), respectively $\left(\chi^{2} P<0.01\right)$ (Figure 2A and Supplemental Table 1 and Supplemental Table 6). This analysis further revealed a group of downregulated proteins with concomitant decrease in whole-gene mRNA (Figure 2B, red box) and another whose wholegene mRNA was not significantly changed (Figure 2B, blue box). This suggests mutant SF3B1 may downregulate some proteins by direct suppression of mRNA (i.e., through missplicing-induced NMD) and others through translational or posttranslational mechanisms. Indeed, among downregulated and misspliced proteins, $27 \%$ (14/51) showed evidence of likely suppression by NMD, with reduction in whole-gene mRNA expression and aberrant junctions predicted to be NMD-sensitive (Figure 2, A and B, and Supplemental Table 6), and another 49\% (25/51) had unchanged 
A

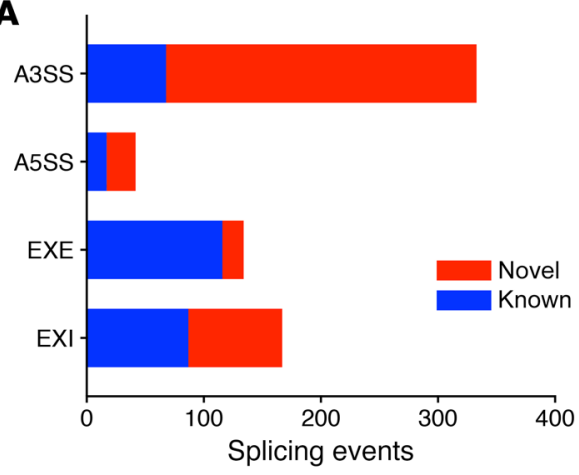

B

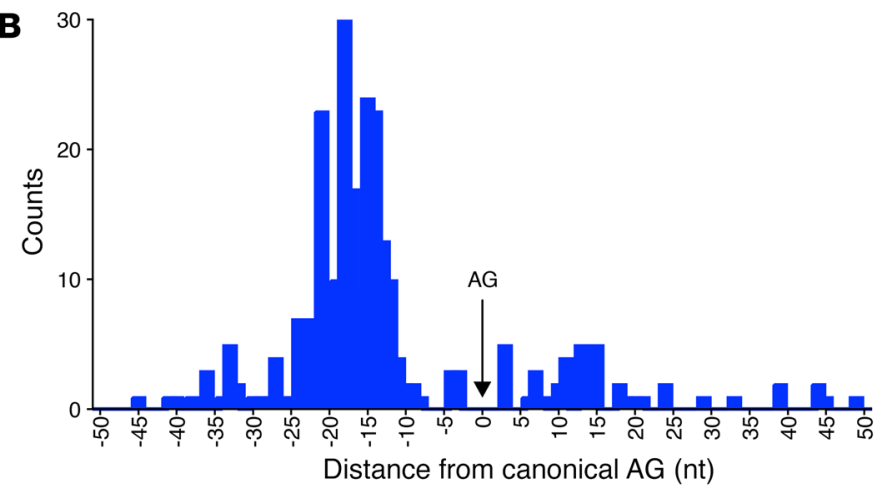

C

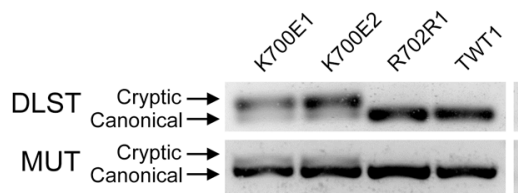

D

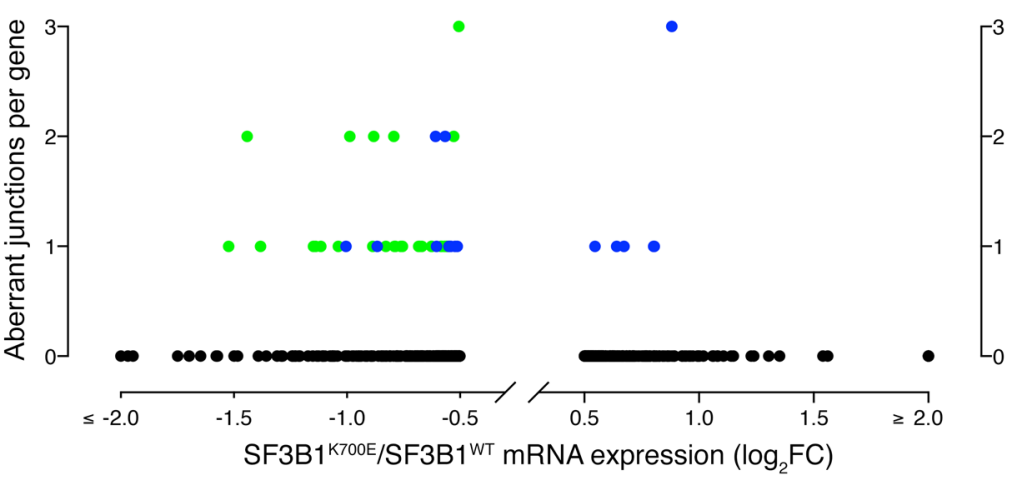

E Junctions upregulated in SF3B1 $1^{\text {MUT }}$

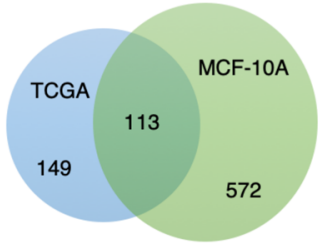

Genes downregulated in SF3B1 ${ }^{\text {MuT }}$

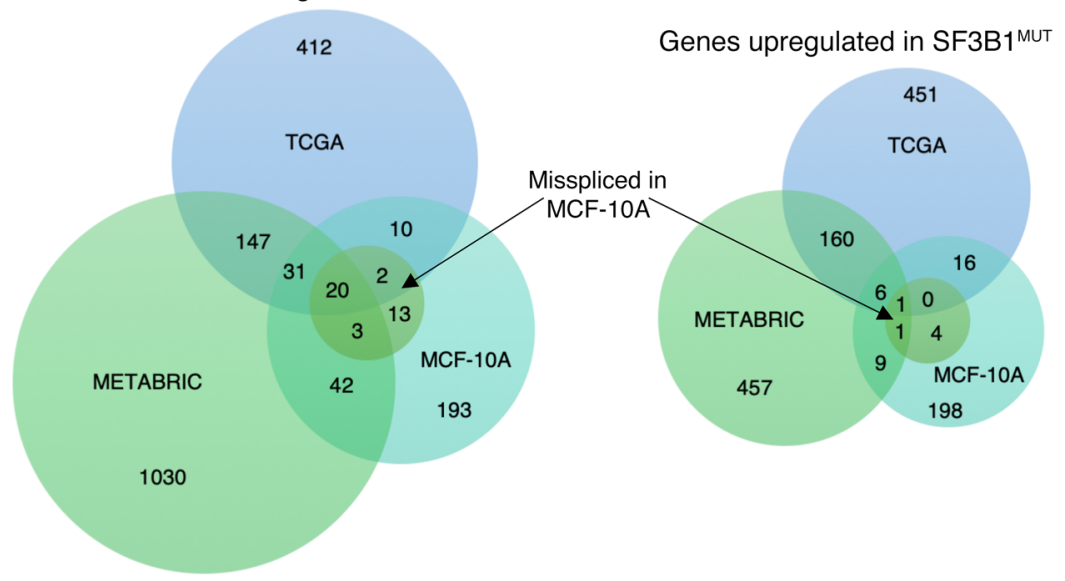

Figure 1. Mutant SF3B1 alters the transcriptome of breast epithelial cells. (A) Classification of splice junctions upregulated in SF3B $7^{K 700 E}$ MCF$10 \mathrm{~A}$ cells. A3SS, alternate $3^{\prime}$ splice site; A5SS, alternate 5' splice site; EXI, exon inclusion; EXE, exon exclusion. Known refers to junctions annotated, and novel refers to those not annotated, in transcriptional databases of normal human tissue. (B) Nucleotide position of cryptic 3' splice sites, relative to canonical AG, for all aberrant junctions in SF3B $7^{\text {K700E }}$ MCF-10A cells. (C) PCR validation of missplicing of the MUT and DLST genes in all 4 SF3B ${ }^{\text {MUT }}$ MCF-10A clones. Data are representative of 2 independent experiments. (D) Number of aberrant junctions per gene plotted against whole-gene mRNA expression in isogenic MCF-10A cells. Green, at least one NMD-sensitive junction; blue, NMD-insensitive junctions. (E) Transcriptional overlap between SF3B1MUT human breast tumors in TCGA and METABRIC data sets and SF3B ${ }^{\text {K700E }}$ MCF-10A cells. Overlap of aberrant junctions (upper) and whole-gene mRNA expression (lower) are shown. The smaller circles contain those genes with both altered whole-gene expression and aberrant junctions in SF3B ${ }^{\text {KTOOE }}$ MCF-10A cells. 
A

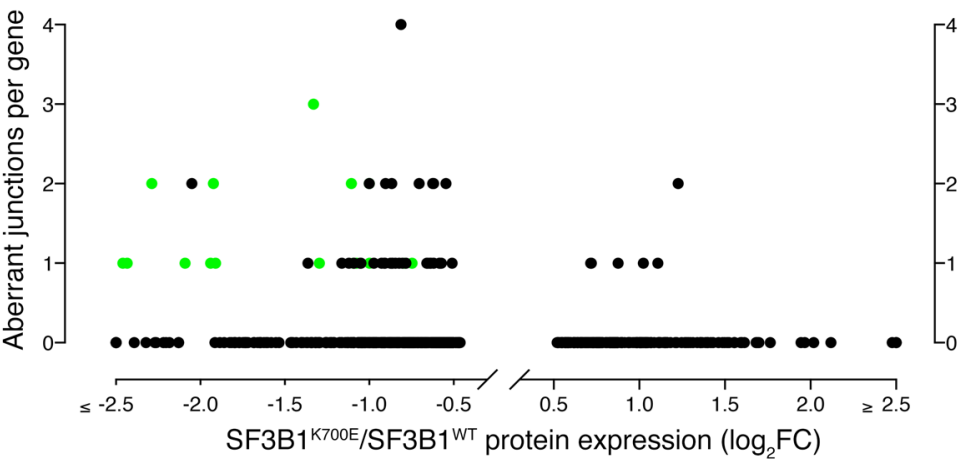

B

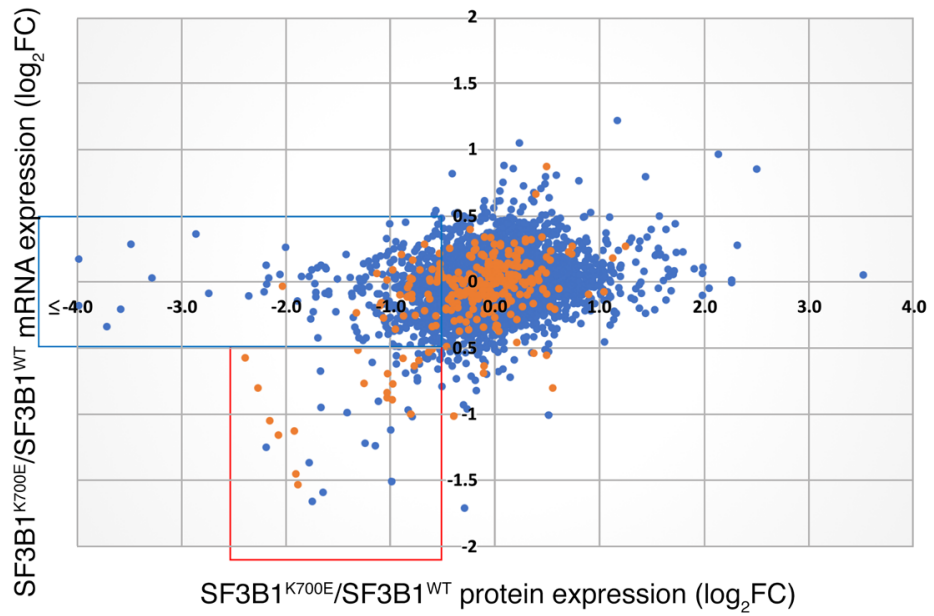

E

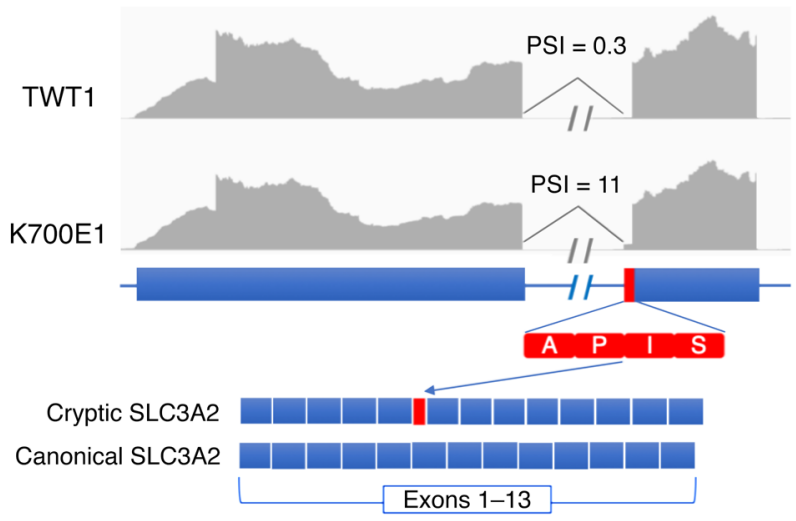

$\mathbf{F}$

Purine ribonucleoside monophosphate metabolic process Mitochondrial electron transport, ubiquinol to cytochrome c

Generation of precursor metabolites and energy Mitochondrial ATP synthesis coupled electron transport Purine ribonucleoside triphosphate metabolic process Regulation of TOR signaling
Regulation of reactive oxygen species metabolic process Hydrogen ion transmembrane transport Unsaturated fatty acid metabolic process Aerobic respiration

Epidermal growth factor receptor signaling pathway Cellular response to insulin stimulus GDP metabolic process Protein localization to kinetochore Protein localization to chromosome, telomeric region Protein localization to chromosome, telomeric region
Protein localization to chromosome, centromeric region Spliceosomal shRNP assembly RNA splicing

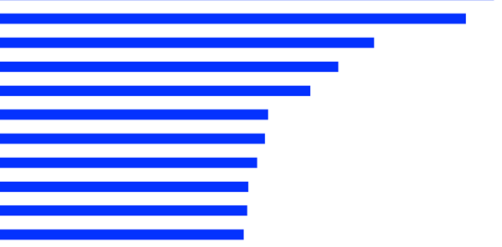

Downregulated Upregulated
D

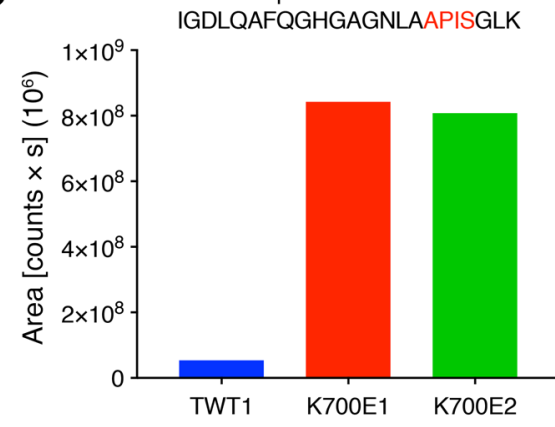

Figure 2. Mutant SF3B1 reshapes the proteome of breast epithelial cells. (A) Aberrant junctions per gene plotted against protein expression determined by SILAC in isogenic MCF-10A cells. Green indicates genes with downregulated whole-gene mRNA and NMD-sensitive junctions. (B) Integration of protein and whole-gene mRNA expression in isogenic MCF-10A cells. Misspliced genes are in orange. The red box shows a population of genes with downregulated mRNA and protein, and the blue box shows a population with downregulated protein, but not mRNA. (C) Western blot validation of protein downregulation for misspliced genes MUT and DLST in isogenic MCF-10A cells. Data are representative of 2 independent experiments (D) Quantification of cryptic SLC3A2 peptide abundance by integration of ion counts from mass spectra in isogenic MCF-10A cells obtained by SILAC. (E) RNA-seq correlation of cryptic splicing of SLC3A2 in isogenic MCF-10A cells, showing insertion of A-P-I-S between exons 5 and 6 . PSI of the cryptic junction is shown. (F) GO pathway enrichment analysis of proteins differentially expressed in SF3B $1^{\text {K700E }}$ MCF-10A cells. 
whole-gene mRNA expression and aberrant junctions predicted to be NMD-insensitive (Figure 2, A and B, and Supplemental Table 6). For the few proteins upregulated and misspliced, whole-gene mRNA was not increased, suggesting translational or posttranslational mechanisms may be at work (Figure 2, A and B, and Supplemental Table 7). Importantly, we orthogonally validated candidate protein alterations with Western blotting in both our MCF-10A and hTERT-IMEC isogenic models (Figure 2C and Supplemental Figure 2B). These data indicate that the predominant association of missplicing by $S F 3 B 1^{M U T}$ with down, rather than up, regulation of misspliced genes observed at the transcript level also extends to the proteome in breast epithelial cells.

Because SILAC quantifies the most abundant fraction of the proteome-and because the splicing pattern of most genes is a single dominant isoform and several less abundant minor onesthe above analysis in fact reflects how mutant $S F 3 B 1$ affects the expression of dominant protein isoforms $(18,19)$. We therefore next sought to determine whether any known or novel alternative splice junctions upregulated by mutant $S F 3 B 1$ produce corresponding junction-spanning peptides. Known alternative splice isoforms of any kind could only be delineated in $2.5 \%(141 / 5,715)$ of our identified proteins, and this set of isoforms did not happen to contain any of the known junctions upregulated by mutant $S F 3 B 1$. We did, however, find one peptide that spanned a novel cryptic 3' splice acceptor site in the heavy chain of the integrin protein CD98, also known as SLC3A2. In each $S F 3 B 1^{K 700 E}$ clone, abundance values of this cryptic peptide were 16-fold higher than the low-level expression in control cells (Figure 2D). The protein change was an insertion of 4 amino acids (A-P-I-S), and RNA-seq data showed the corresponding cryptic transcript resulting from insertion of the 12-bp intronic sequence preceding exon 5 that encodes these residues (Figure 2E). Mirroring the peptide-level quantification, the percent spliced in (PSI) of this cryptic splice junction was 34 -fold higher (11 vs. $0.3 \%, P$ value $\left.=5.89 \times 10^{-9}\right)$ in $S F 3 B 1^{K 700 E}$ cells (Figure $2 \mathrm{E}$ ). Thus, cryptic protein can be produced in breast epithelial cells following physiologic, heterozygous expression of mutant SF3B1.

Lastly, we subjected all protein expression changes, whether associated with direct missplicing or not, to Gene Ontology analysis. Among proteins downregulated in $S F 3 B 1^{K 700 E}$ cells, there was a notable enrichment of pathways involved in energy metabolism, while upregulated proteins showed enrichment of pathways involving mRNA processing, mRNA splicing, and chromosomal localization of proteins (Figure 2F). Together, these data show that mutant $S F 3 B 1$ reshapes the proteome in breast epithelial cells, producing missplicing-associated gene downregulation, cryptic protein, and altered expression of proteins in multiple cellular pathways.

Evidence for reprogramming of mitochondrial metabolism by $S F 3 B 1^{M U T}$ in breast epithelial cells. Given the contextual robustness of missplicing-associated gene downregulation induced by mutant $S F 3 B 1$, we next examined the metabolic pathways enriched in proteins downregulated in $S F 3 B 1^{M U T}$ cells. One notably specific signal was in the ubiquinol-cytochrome c oxidoreductase complex, also known as mitochondrial complex III of the electron transport chain (ETC). It is formed by assembly factors UQCC1 and UQCC2, which stabilize mitochondrial cytochrome B (MT-CYB) and deliver it to other ubiquinol-cytochrome $\mathrm{c}$ reductase proteins to form mature complex III (Figure 3A) (20, 21). Among the 50 most downregulated proteins in our SILAC experiment, 7 were part of this complex (Figure 3A). None of these 7 had $S F 3 B 1^{M U T}$-dependent changes in whole-gene mRNA expression (Supplemental Table 2), suggesting their downregulation was at the protein level and not a direct result of missplicing. However, the assembly factor UQCC1 was heavily misspliced, with multiple aberrant transcripts containing cryptic terminal exons (Figure 3B). Though truncated, these aberrant transcripts would be NMD-insensitive, and their presence likely explains why the whole-gene mRNA expression (i.e., the sum of all isoforms) of UQCC1 was not lower by RNA-seq. Yet full-length, canonical UQCC1 transcript was indeed lower in $S F 3 B 1^{K 700 E}$ cells (Figure 3B), consistent with a zero-sum competition model of splicing between U2 snRNP complexes with WT or mutant SF3B1 (9). UQCC1 was not a protein captured by SILAC, but Western blotting revealed it to be decreased in $S F 3 B 1^{K 700 E}$ cells, along with other complex III proteins (Figure 3C). We then constitutively expressed canonical UQCC1, and this rescued not only UQCC1 levels but also levels of other complex III proteins in $S F 3 B 1^{K 700 E}$ MCF-10A cells (Figure 3C). UQCC1 and UQCC2 were also decreased by $S F 3 B 1^{\text {K7OOE }}$ in hTERT-IMEC cells, although the downregulation of mature complex III proteins was less pronounced, perhaps due to a greater baseline reserve of the UQCC1 assembly factor in this cell line (Supplemental Figure 3). SF3B1 ${ }^{\text {MUT }}$ breast cancers from TCGA also did not show reduced whole-gene UQCC1 expression by RNA-seq, but they did by microarray analysis, whose probes capture canonical, but not cryptic, mRNA (Supplemental Figure 4). UQCC1 was also the single most downregulated gene in $S F 3 B 1^{M U T}$ tumors in the METABRIC data set, which used a microarray platform for mRNA analysis (Supplemental Figure 4). These data show that physiologic expression of mutant $S F 3 B 1$ is sufficient to downregulate mitochondrial complex III levels, likely through missplicing of UQCC1.

In transferring electrons from ubiquinol to cytochrome c, complex III fuels oxidative phosphorylation as well as the regeneration of electron acceptors for aspartate biosynthesis $(22,23)$. We therefore sought to determine whether the observed $S F 3 B 1^{M U T}$-induced protein changes are accompanied by metabolic alterations. We first performed targeted metabolomics using liquid chromatography electrospray ionization tandem mass spectrometry (LC-ESI-MS/ MS) in our isogenic cells, quantifying energy-relevant amino acids and metabolites involved in glycolysis and the TCA cycle. We validated the metabolomics platform using cells exposed to the biguanide metformin, which induced several known metabolic consequences of this drug in control MCF-10A cells, including increased lactate, $\alpha$-ketoglutarate, and serine, along with decreased citrate, succinate, and aspartate (24). Interestingly, mutant SF3B1 did induce multiple alterations in energy metabolites, including decreases in the TCA cycle metabolites succinate, fumarate, and malate; increases in glucose and 3-hydroxybutyrate; and decreases in amino acids such as serine, glycine, and aspartate (Figure 3D). For orthogonal validation, we confirmed decreased aspartate in an independent $S F 3 B 1^{K 700 E}$ MCF-10A clone using a fluorometric assay (Supplemental Figure 5). While attributions of metabolite abundance to particular protein expression levels are made difficult by the complex interplay of metabolite and enzyme dynamics (25), the finding of decreased aspartate in $S F 3 B 1^{M U T}$ cells may nonetheless be suggestive of ETC impairment, as aspartate biosynthesis is 
A

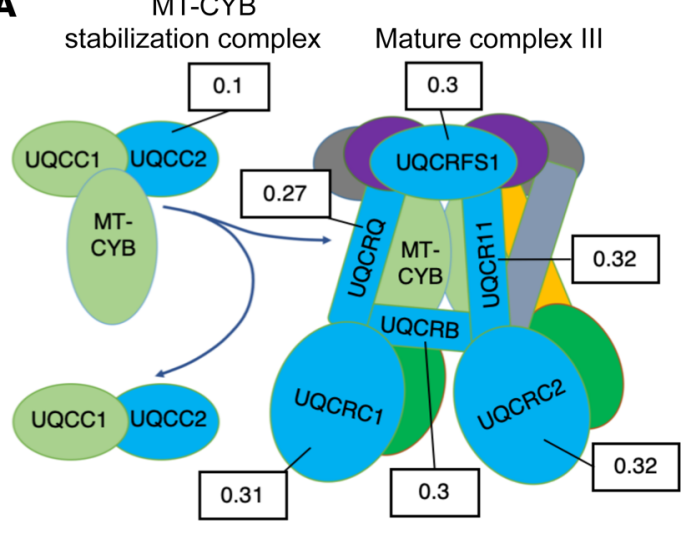

C

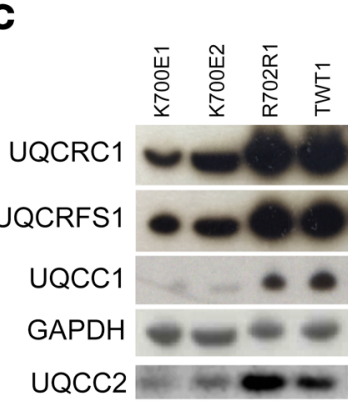

GAPDH
MT-CYB

abilization complex

Mature complex III

(1)

0.3

\section{UQCRFS1}

$\beta$-Actin

UQCC2

UQCC1

GAPDH

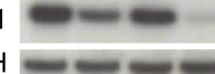

B
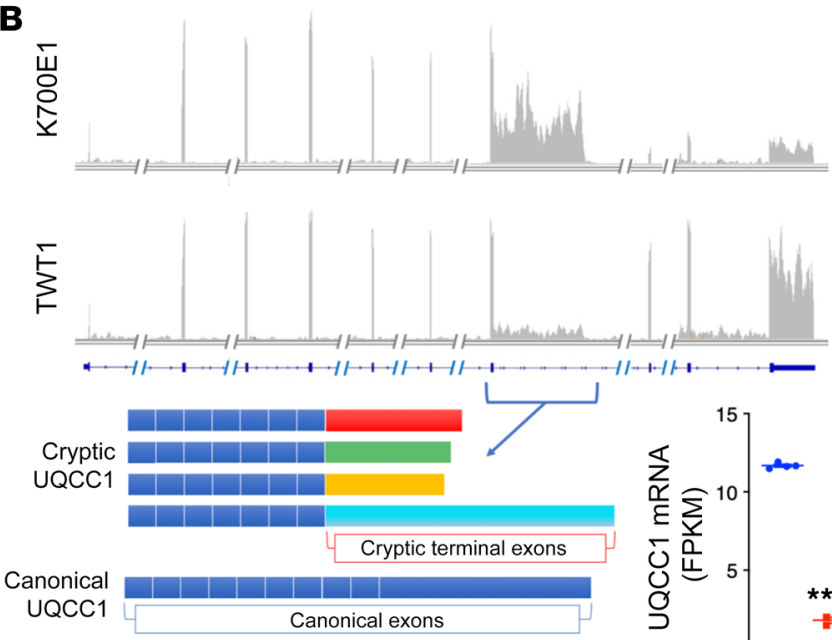

D $\mathrm{R} 702 \mathrm{R} 1$

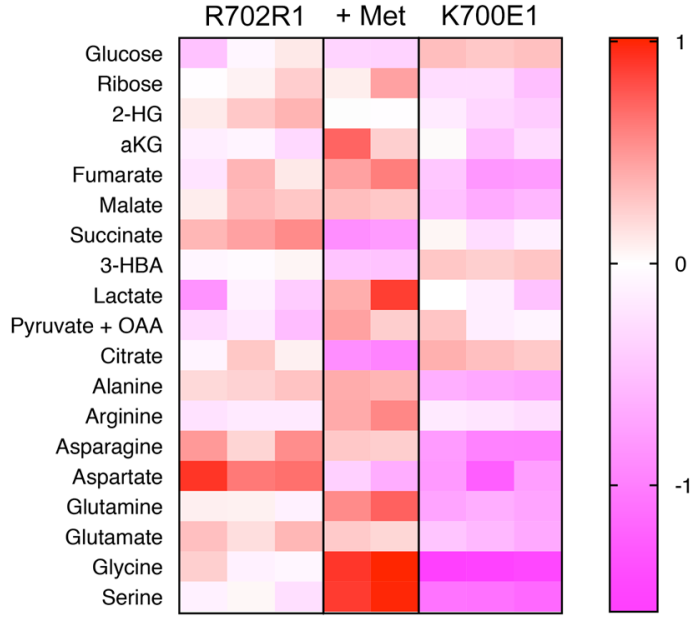

E

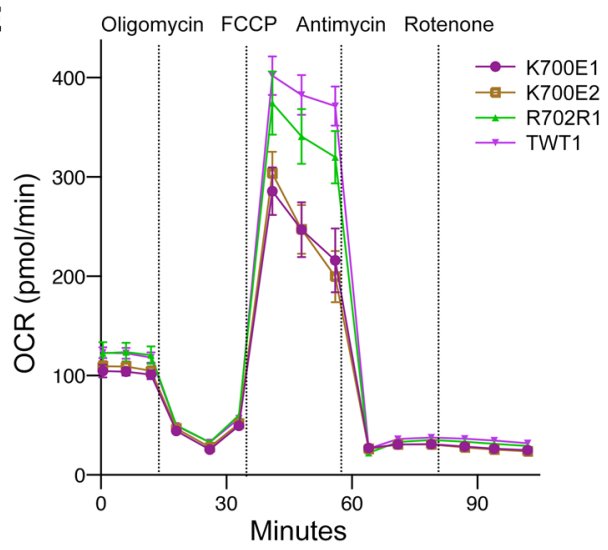

Figure 3. Evidence for reprogramming of mitochondrial metabolism in SF3B1 ${ }^{\text {UUT }}$ cells. (A) Complex III assembly. Blue proteins were captured by SILAC in isogenic MCF-10A cells, with SF3B1 MUT $/ S F 3 B 1^{W T}$ abundance ratios labeling them. (B) RNA-seq from isogenic MCF-10A cells shows UOCC1 is misspliced by $S F 3 B 1^{M U T}$ (upper), creating transcripts with cryptic terminal exons (lower left). Canonical, but not whole-gene, UOCC1 mRNA is reduced in RNA-seq data (lower right). ${ }^{* * *} P<0.0001$ for $t$ test comparing canonical FPKM in all mutant to all WT. (C) Western blotting shows downregulated complex III in isogenic MCF-10A cells (left), and canonical UOCC1 overexpression rescues complex III levels in SF3B1 ${ }^{M U T}$ cells (right). Vec, vector. Data are representative of 2 independent experiments. Upper left panel represents the same membrane previously blotted for MUT and DLST in Figure 2C. (D) Metabolite quantification in isogenic MCF-10A cells. Three biological replicates were used in R702R1 and K700E2 cells, 2 replicates for R702R1 + metformin (Met), and Z scores are shown. (E) Oxygen consumption rate (OCR) in isogenic MCF-10A cells as measured by a Seahorse XF Analyzer. Data are representative of 2 independent experiments, and 6 biologic replicates per conditions were used.

an essential function of mitochondrial respiration in multiple cell contexts $(22,23,26-28)$. To test mitochondrial respiration directly, we measured oxygen consumption rate in 2 mutant and 2 WT MCF10 A clones with a Seahorse XF Analyzer, and this demonstrated that both basal and maximal cellular respiration were decreased by
$S F 3 B 1^{M U T}$ (Figure 3E and Supplemental Figure 6). In contrast, extracellular acidification rate was unaffected (Supplemental Figure 6). Together, these results provide evidence that $S F 3 B 1^{M U T}$ induces a reprogramming of mitochondrial metabolism characterized by a decreased capacity for cellular respiration in breast epithelial cells. 
A

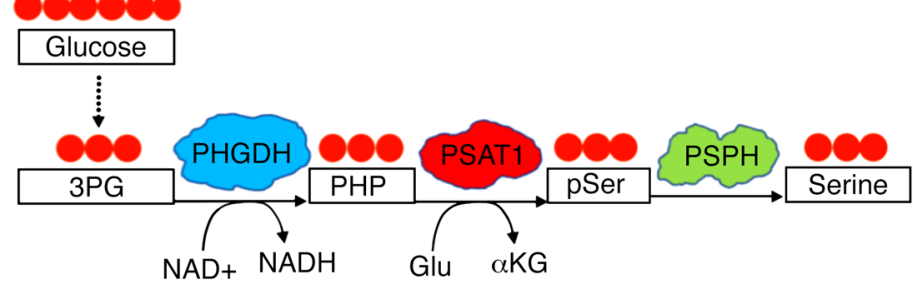

B
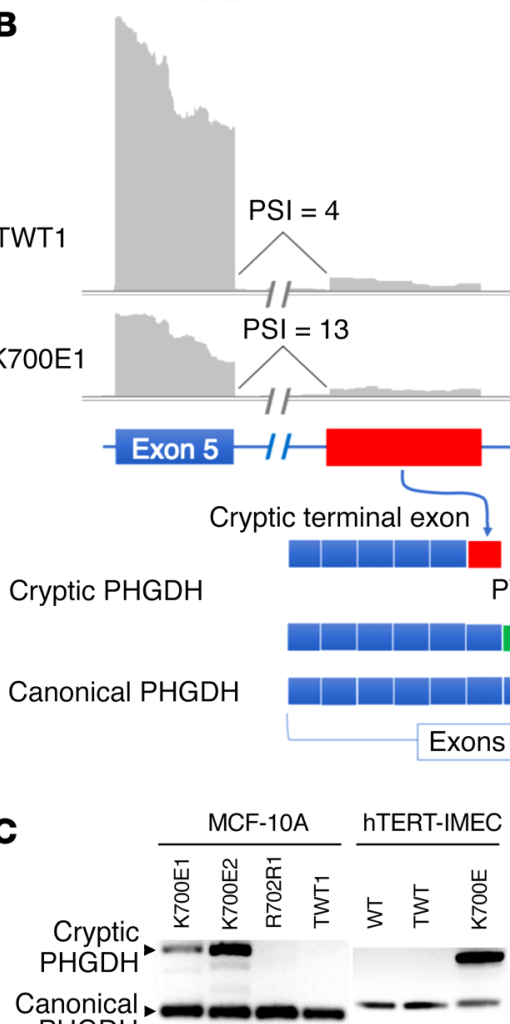

PHGDH
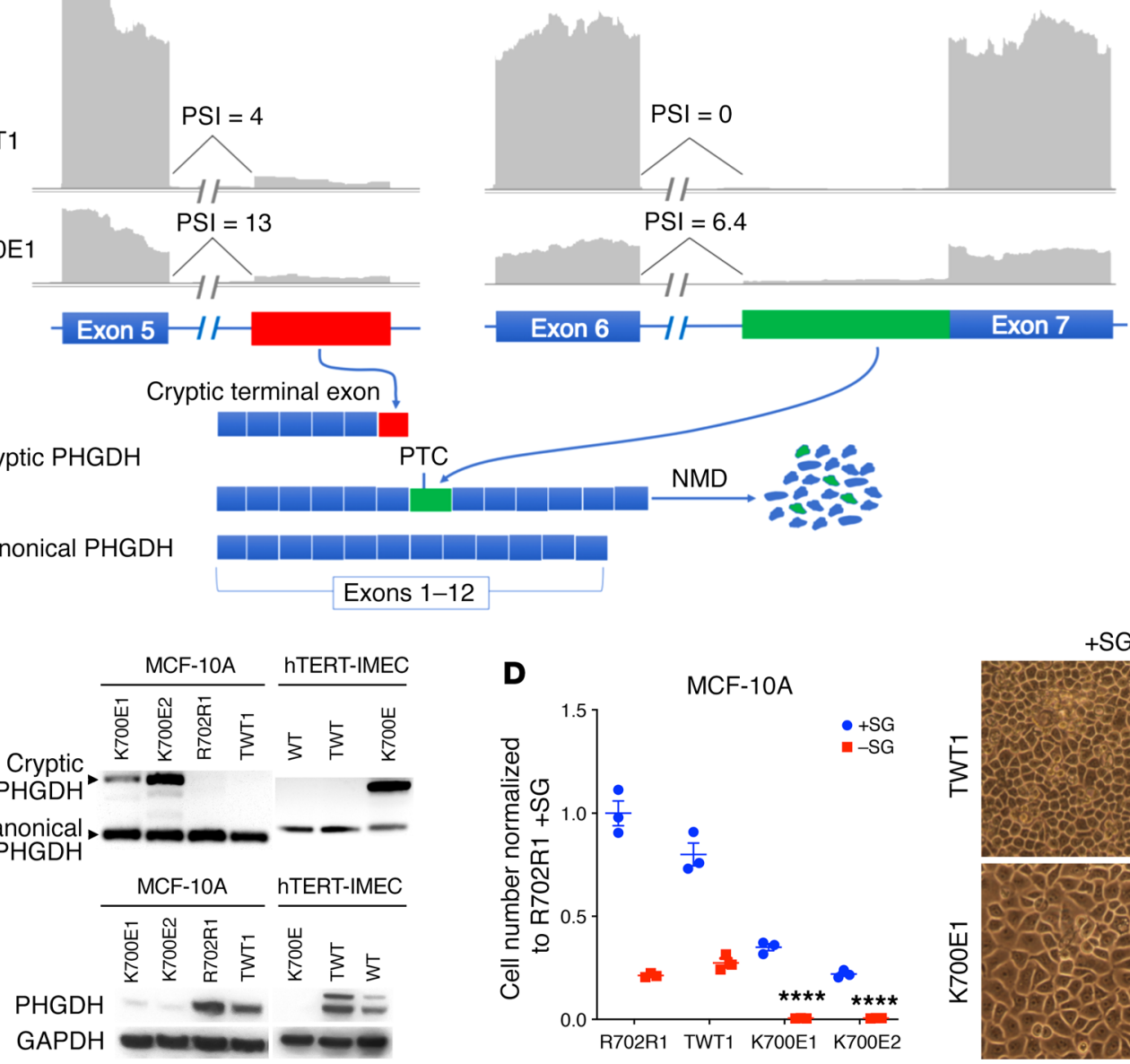
Mutant SF3B1 suppresses the serine synthesis pathway in breast epithelial cells. Another metabolic gene whose expression was strikingly affected by $S F 3 B 1^{M U T}$ was phosphoglycerate dehydrogenase $(P H G D H)$, an enzyme that catalyzes the conversion of 3-phosphoglycerate to phosphohydroxypyruvate as the rate-limiting step in de novo synthesis of the nonessential amino acid serine (Figure 4A). SF3B1 ${ }^{\text {MUT }}$ knockin breast epithelial cells exhibited enrichment of 2 different aberrant splice junctions in $P H G D H$, which are predicted to decrease total $P H G D H$ mRNA expression by creating a cryptic terminal exon and inducing NMD, respectively (Figure 4, B and C). Indeed, whole-gene mRNA expression was lower by RNA-seq in SF3B1 ${ }^{M U T}$ MCF-10A (Supplemental Table 2), $P H G D H$ protein was among the most downregulated proteins captured by SILAC (Supplemental Table 6), and Western blotting confirmed decreased $P H G D H$ protein in both $S F 3 B 1^{M U T}$ MCF-10A and hTERT-IMEC cells (Figure 4C). Moreover, in the TCGA and METABRIC data sets, $P H G D H$ was among the most downregulated genes (Supplemental Table 4) in $S F 3 B 1^{M U T}$ tumors.

Previous studies have shown that deliberate suppression of PHGDH or other components of the serine synthesis pathway renders mammalian cells less capable of growth and survival without exogenous serine, or, as is usually done in such studies, serine and glycine (SG), since serine can be synthesized directly from glycine (29-31). We therefore asked whether $S F 3 B 1^{\text {MUT }}$ cells, with suppressed $P H G D H$ levels, might exhibit a dependence on exogenous SG. When cultured in media without SG (-SG media), WT MCF-10A cells grew more slowly than in complete media, but cells nonetheless remained viable and mitotic (Figure 4D). In contrast, $S F 3 B 1^{K 700 E}$ cells showed minimal growth without exogenous serine and glycine (Figure 4D). For hTERT-IMECs, WT cells were naturally more capable of growing without SG than WT MCF-10As, yet $S F 3 B 1^{K 700 E}$ hTERT-IMECs still showed minimal growth without SG in both short-term proliferation and long-term colony formation assays (Figure 4E). Of note, the baseline growth in full media of $S F 3 B 1^{K 700 E}$ breast epithelial cell knockins was slower than that of control cells (Supplemental Figure 7), but SG starvation disproportionately suppressed growth from this lower baseline in $S F 3 B 1^{K 700 E}$ cells (Figure 4, D and E). These data demonstrate that $S F 3 B 1^{M U T}$ increases dependence on exogenous serine and glycine for growth in breast epithelial cells.

PHGDH downregulation and serine/glycine auxotrophy suggest that serine synthesis could be impaired by $S F 3 B 1^{M U T}$. Indeed, our metabolomic analysis of isogenic MCF-10A cells did show decreased total intracellular serine and glycine levels in $S F 3 B 1^{K 700 E}$ cells (Figure 3D). However, while suppression of the serine synthesis pathway decreases total intracellular serine and glycine in certain cell types grown in regular media, in most cell types suppression of the serine synthesis pathway will not decrease total intracellular serine and glycine unless the exogenous supply of serine is restricted $(30,32,33)$. In contrast, the fraction of serine synthesized de novo in cells, as revealed by isotope tracing of ${ }^{13} \mathrm{C}$-glucose into $\mathrm{M}+3$ serine, is ubiquitously decreased by suppression of the serine synthesis pathway in different cell types (33-35). Therefore, to directly determine whether serine synthesis is decreased by $S F 3 B 1^{M U T}$, we performed isotope tracing of ${ }^{13} \mathrm{C}$-glucose in our isogenic hTERT-IMEC cells in both +SG and -SG conditions. The total serine was lower in $S F 3 B 1^{K 700 E}$ hTERT-IMEC cells at baseline compared with $S F 3 B 1^{W T}$ cells, with a fold change of 0.581 (Supplemental Figure 8 and Supplemental Table 9), similar to $S F 3 B 1^{\text {MUT }}$ MCF-10A cells with a fold change of 0.497 (Supplemental Table 8). In media containing SG (+SG media), the percentage of $\mathrm{M}+3$ serine synthesized from ${ }^{13} \mathrm{C}$-glucose in $S F 3 B 1^{W T}$ hTERT-IMEC cells was $2.7 \%$ of the total serine, comparable to baseline serine synthesis rates in regular media of breast epithelial cells seen in other studies (Figure 4F, Supplemental Figure 8, and Supplemental Table 9) $(36,37)$. In contrast, the percentage of $M+3$ serine in SF3B1 ${ }^{K 700 E}$ hTERT-IMECs was $0.19 \%$ of the total serine in + SG media, equal to only $4 \%$ of the $\mathrm{M}+3$ serine in $S F 3 B 1^{W T}$ cells (Figure 4F, Supplemental Figure 8, and Supplemental Table 9). Upon starvation of serine and glycine, both the absolute total serine and absolute M+3 serine were decreased compared with +SG conditions in $S F 3 B 1^{W T}$ cells, but the percentage of total serine made up by $\mathrm{M}+3$ serine increased to $77 \%$, consistent with previous work on serine isotope changes upon SG starvation (Figure 4F, Supplemental Figure 8, and Supplemental Table 9) $(38,39)$. In contrast, the absolute total serine decreased even further in $S F 3 B 1^{K 700 E}$ cells in -SG media, to $20 \%$ that of $S F 3 B 1^{W T}$ cells, and the $\mathrm{M}+3$ serine fraction comprised only $2.6 \%$ of the total, representing $0.7 \%$ of the $\mathrm{M}+3$ serine in $S F 3 B 1^{W T}$ cells in -SG conditions (Figure $4 \mathrm{~F}$, Supplemental Figure 8, and Supplemental Table 9). These data indicate that de novo serine synthesis is severely impaired in $S F 3 B 1^{\text {K7OOE }}$ breast epithelial cells, and the inability of these cells to adequately generate flux through the serine synthesis pathway likely contributes to their growth impairment in -SG conditions.

Transformed SF3B1 ${ }^{M U T}$ cells exhibit PHGDH downregulation and vulnerability to serine starvation. Deprivation of serine and glycine has now been shown to suppress growth of certain cancers in vitro and in vivo, introducing a novel anticancer treatment strategy (24, 26-28). We therefore asked whether $S F 3 B 1^{M U T}$ induces $P H G D H$ downregulation and vulnerability to serine starvation in fully transformed human cancer cells. In breast cancer, $S F 3 B 1$ mutations occur predominantly in hormone receptor-positive tumors, so we utilized an $S F 3 B 1^{K 70 O E}$ knockin of ER-positive T47D breast cancer cells. As in untransformed breast epithelial cells, $P H G D H$ mRNA was misspliced and its protein was downregulated by $S F 3 B 1^{K 700 E}$ in T47D cells (Figure 5A). We then assayed the 3D growth of these isogenic cells in conditions with and without SG. Notably, SF3B1 ${ }^{\text {K7OOE }}$ T47D cells showed no significant growth deficit in regular media compared with $S F 3 B 1^{W T}$ cells (Figure $5 \mathrm{~A}$ ), in contrast to the baseline slower growth exhibited by untransformed $S F 3 B 1^{M U T}$ breast epithelial cells (Supplemental Figure 7). Additionally, the growth of WT T47D cells was not significantly impaired by SG starvation in these conditions, indicating adequate de novo serine synthesis capacity in these cells (Figure 5A). In contrast, the size of $S F 3 B 1^{K 700 E} \mathrm{~T} 47 \mathrm{D}$ spheroids was reduced by $30 \%-40 \%$ in -SG media in 2 independent knockin clones, as compared with those grown in +SG, demonstrating that $S F 3 B 1^{M U T}$ induces vulnerability to serine and glycine starvation in fully transformed breast cancer cells (Figure 5A).

Given that mutation-targeting cancer treatments are beginning to transcend the traditional boundaries of tissue of origin in their applications (40), we next sought to determine whether $S F 3 B 1^{M U T}$ cancer cells from other tissue types might exhibit this vulnerability. SF3B1 mutations are more common in hematologic malignancies than in solid tumors, and we found that $P H G D H$ 
A

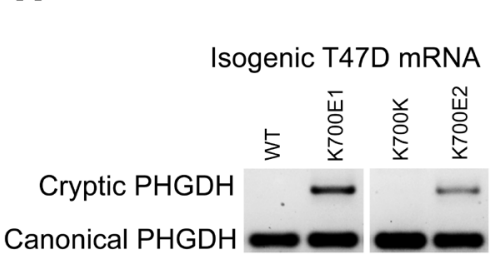

Isogenic T47D protein

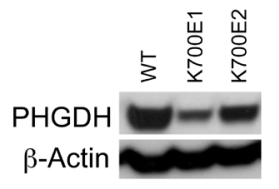

B

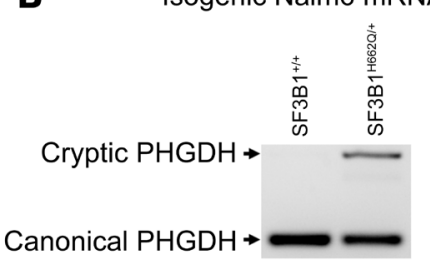

C

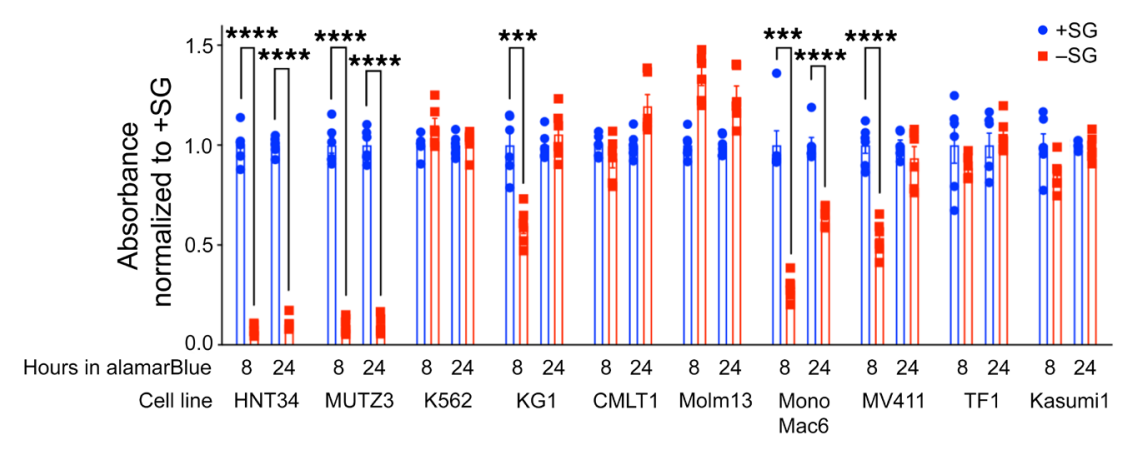

SF3B1 status K700E K666N
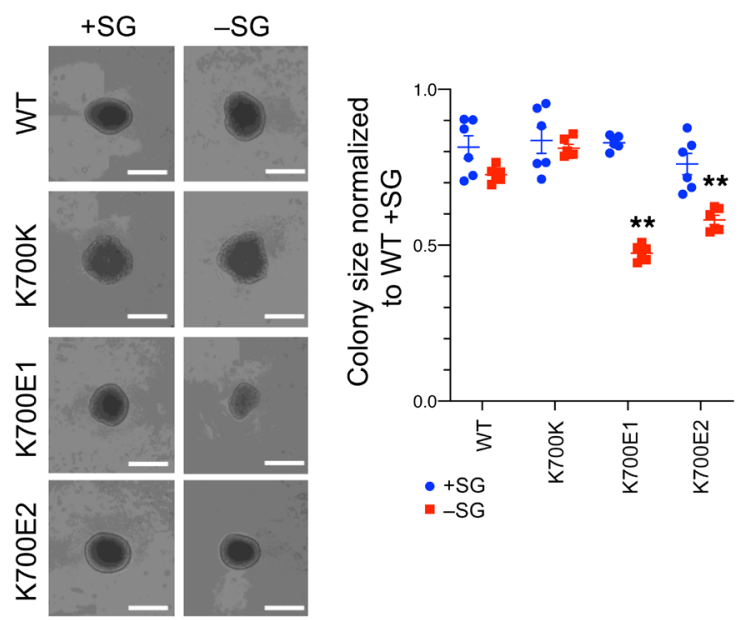

Isogenic Nalm6 protein

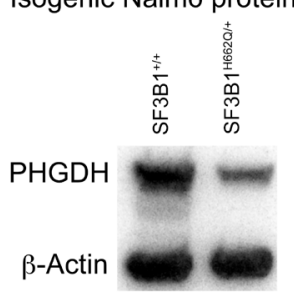

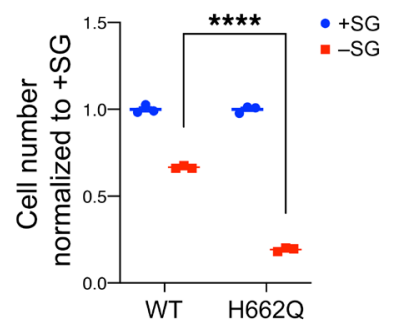

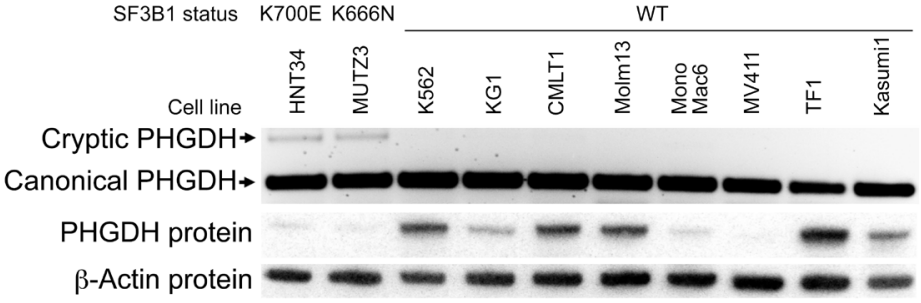

expression was downregulated in $S F 3 B 1^{M U T}$ cases of myelodysplastic syndrome and acute myeloid leukemia in published gene expression data sets of these diseases (Supplemental Figure 9) (41, 42). We then interrogated the effect of SG starvation on Nalm6 leukemia cells with knockin of the $S F 3 B 1^{H 662 Q}$ hotspot mutation (9). Compared with WT Nalm6 cells, $S F 3 B 1^{H 662 Q}$ cells exhibited aberrant splicing of $\mathrm{PHGDH}$, lower expression of $\mathrm{PHGDH}$ protein, and a heightened sensitivity to SG starvation (Figure $5 \mathrm{~B}$ ). We then turned to a panel of leukemia cell lines with or without native $S F 3 B 1$ mutations. In 8 leukemia lines WT for $S F 3 B 1$, all demonstrated growth and viability over 4 days without SG, though with variable amounts of partial growth suppression (Figure $5 \mathrm{C}$ ).
Figure 5. SF3B1 ${ }^{M U T}$ cancer cells show missplicing-associated downregulation of PHCDH and vulnerability to SG starvation. (A) T47D breast cancer cells with knockin of SF3B ${ }^{\text {K700E }}$ show aberrant $P H G D H$ splicing (upper left), decreased PHGDH protein (lower left), and decreased 3D colony growth in Matrigel (middle and right). Scale bars are $1 \mathrm{~mm}$. Colony counts are from 6 biologic replicates per cell line and data are from 2 independent experiments. ${ }^{*} P<$ 0.01 for $t$ test comparing all mutant to all WT, but independent clones are also shown. (B) SF3B1 16620 Nalm6 knockins show aberrant $P H C D H$ splicing (left), decreased PHGDH protein (middle), and lower relative growth in -SG (right). Cell counts are from 3 biologic replicates per condition, and data are representative of 3 independent experiments. ${ }^{* * *} P<$ 0.0001 for $t$ test compared with - SC for WT. (C) Leukemia cell lines with native SF3B1 mutation show PHGDH missplicing, low $P H C D H$ protein, and increased sensitivity to SG starvation. Absorbance of alamarBlue reaction product was quantified in -SG relative to +SG control after 8 and 24 hours of incubation in the viability reagent. Six biologic replicates were used for alamarBlue, and data are representative of 2 independent experiments. ${ }^{* *} P<0.001$; ${ }^{* * *} P<0.0001$ for $t$ test comparing -SG with $+\mathrm{SG}$.
In contrast, $S F 3 B 1^{K 700 E}$ HNT34 cells and $S F 3 B 1^{K 666 N}$ MUTZ3 cells were nonviable after 4 days without SG, and both cell lines showed aberrant splicing and decreased protein levels of $\mathrm{PHGDH}$ (Figure $5 \mathrm{C})$. We also confirmed deprivation of serine is principally responsible for the suppressive effects of SG starvation in these cells (Supplemental Figure 10). Interestingly, a few SF3B1-WT leukemia cell lines also had lower PHGDH expression (MV411, Monomac-6, KG-1), and these cells did show greater growth suppression without SG than the other SF3B1-WT cell lines (Figure 5C). This suggests that downregulation of $P H G D H$ by mechanisms other than missplicing by mutant $S F 3 B 1$ may also predict for reliance on extracellular serine. Taken together, these data show that in both 


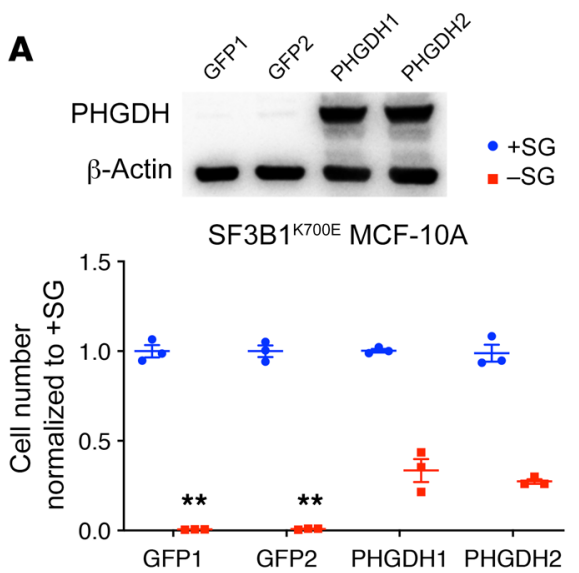

B
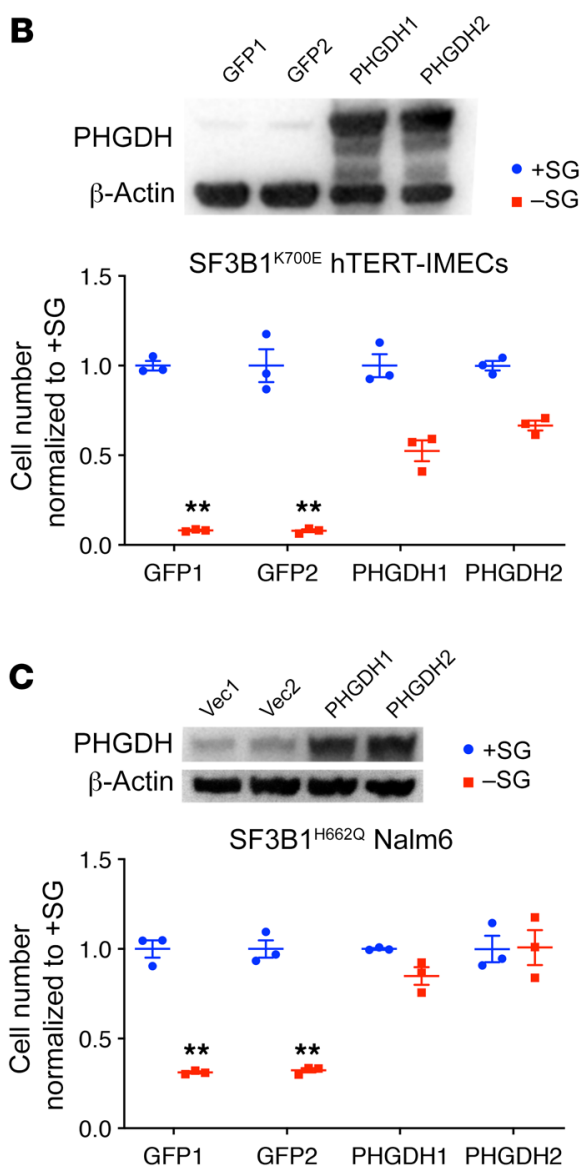
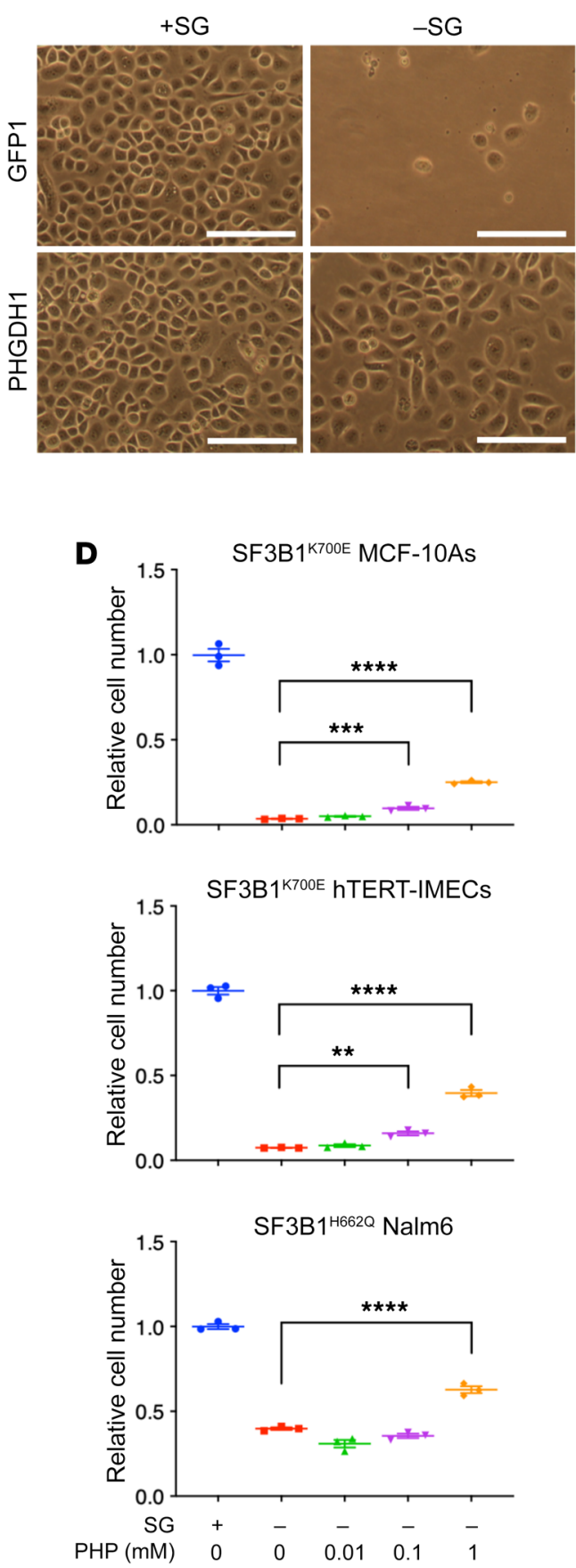

Figure 6. PHCDH overexpression and PHP rescue growth without SG in SF3B1 ${ }^{\text {MUT }}$ cells. (A) Western blot of $P H G D H$ levels in independent polyclona pools of $S F 3 B 7^{\text {K700E }}$ MCF-10A cells with overexpressed GFP or PHGDH (upper left). Quantification of growth of overexpression populations in media with or without SC (lower left) and representative images (right). Scale bars: 100 $\mu \mathrm{m}$. (B) PHGDH Western blot in GFP- or PHCDH-overexpressed populations of SF3B $7^{\text {KOOOE }}$ hTERT-IMEC cells (upper), and quantification of their growth in media with or without SC (lower). (C) Western blot of PHGDH in SF3B ${ }^{H 6620}$ Nalm6 cells after overexpression of vector or PHGDH (upper), and +SG and -SG growth quantification (lower). (D) Rescue of -SC growth by increasing concentrations of phosphohydroxypyruvate (PHP) in $S F 3 B 7^{M U T}$ cells. All data are representative of 2 independent experiments and 3 biologic replicates per condition were used. ${ }^{* *} P<0.01,{ }^{* *} P<0.001$, ${ }^{* * * *} P<0.0001$ for Dunnett's test comparing all GFP/vector-overexpressed to all PHGDH-overexpressed cells, but independent polyclonal populations are also shown. knockin and naturally mutated cancer cells of multiple cell lineages, SF3B1 mutation is associated with downregulation of $\mathrm{PHGDH}$ and heightened sensitivity to SG starvation.

PHGDH overexpression and PHP supplementation rescue growth without exogenous serine. To determine whether $P H G D H$ downregulation is a mediator of this vulnerability, we reexpressed canonical PHGDH in $S F 3 B 1^{K 700 E}$ breast epithelial cells and $S F 3 B 1^{H 662 Q}$ Nalm6 cells, creating 2 independently derived populations of $P H G D H$-overexpressing cells for each cell model, along with 2 independently derived populations of GFP- or vector-overexpressing controls (Figure 6 and Supplemental Figure 11). In contrast to GFP, $P H G D H$ was able to rescue cell growth without $S G$ in
$S F 3 B 1^{K 700 E}$ MCF-10A cells (Figure 6A). A similar rescue of growth without SG by $P H G D H$ was also seen in both $S F 3 B 1^{K 700 E}$ hTERTIMEC cells and $S F 3 B 1^{H 662 Q}$ Nalm6 cells (Figure 6, B and C). These data support the idea that impaired serine synthesis due to missplicing-associated downregulation of $P H G D H$ contributes to the vulnerability to SG starvation of $S F 3 B 1^{M U T}$ cells. Consistent with this, we also found that growth of SG-starved $S F 3 B 1^{\text {MUT }}$ cells could be rescued by supplementation with phosphohydroxypyruvate (PHP), the reaction product of $P H G D H$ that serves as the substrate for subsequent steps of the serine synthesis pathway (Figure 6D).

Dietary restriction of serine and glycine limit in vivo growth of SF3B1 ${ }^{\text {MUT }}$ tumors. Finally, we sought to determine whether 
A

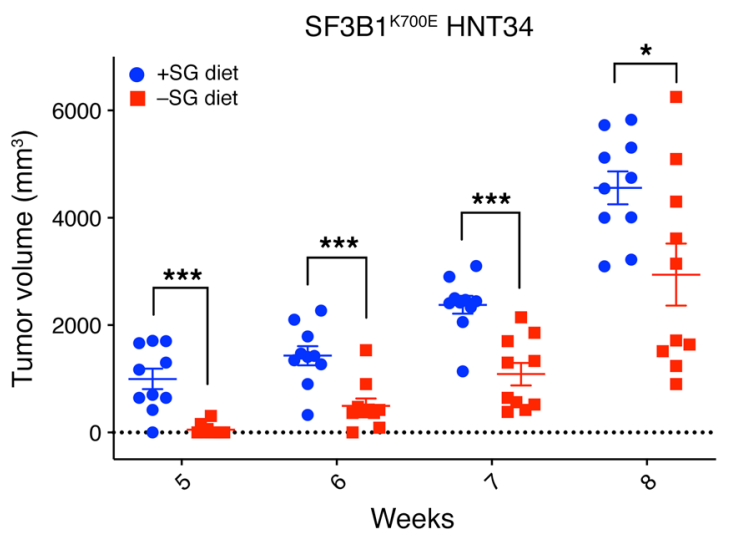

$+S G$

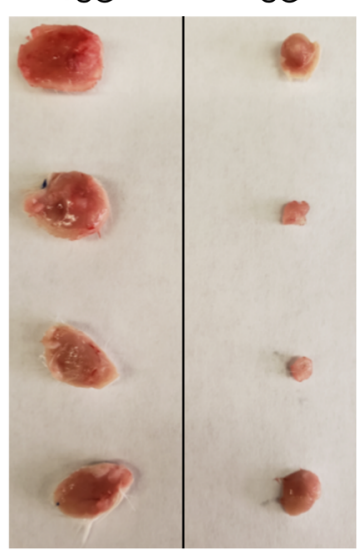

B SF3B1 ${ }^{\mathrm{K} 666 \mathrm{~N}}$ MUTZ3

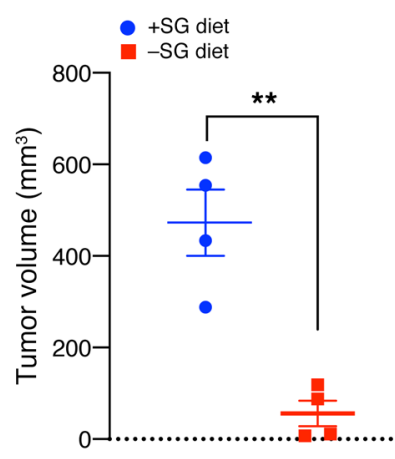

Figure 7. Cancer cells with naturally acquired SF3B1 mutation show vulnerability to serine and glycine starvation in vivo. (A) HNT34 cells were subcutaneously injected into NSG mice given +SG or -SG diets, and tumor volume measurements were obtained starting at 5 weeks, with 10 biologic replicates in each group. (B) MUTZ3 cells were subcutaneously injected into NSGS mice given +SG or -SG diets and tumors were excised from euthanized animals and measured at week 4 , with 4 biologic replicates in each group. ${ }^{* *} P<0.001,{ }^{* *} P<0.01,{ }^{*} P<0.05$ for $t$ tests between dietary groups.

heightened sensitivity to SG starvation in $S F 3 B 1^{M U T}$ cells might be exploited therapeutically in vivo. Vousden and colleagues have shown that mice fed an SG-free diet will achieve a 60\% reduction in blood SG levels, can tolerate the dietary modification, and in certain contexts will exhibit slower tumor growth $(43,44)$. We therefore performed xenograft experiments with $S F 3 B 1^{K 700 E}$ HNT34 cells in mice fed SG-free or amino acid-replete diets. We first employed HNT34 cells both because of their native SF3B1 mutation and because additional in vitro experiments showed their growth could be suppressed by partial serine depletion, a state which reflects the effects of dietary restriction in vivo (Supplemental Figure 12). As shown in Figure 7A, HNT34-derived tumors in mice fed an SG-free diet grew more slowly than those of animals that received a control, SG-containing diet. To determine whether this in vivo inhibitory effect could be seen in other $S F 3 B 1^{M U T}$ cells, we also tested the growth of $S F 3 B 1^{K 666 N}$ MUTZ3 cells. As shown in Figure 7B, the growth of MUTZ3-derived tumors was also decreased in mice fed SG-free food. These data thus provide evidence that SF3B1-mutant cells can be therapeutically targeted in vivo by dietary restriction of serine and glycine.

\section{Discussion}

Here we report that hotspot mutations in the spliceosome protein $S F 3 B 1$ cause a reshaping of the proteome, reprogramming of cellular metabolism, and vulnerability to deprivation of the nonessential amino acid serine. These findings have several novel implications. While previous work has elegantly demonstrated the mechanism by which mutant SF3B1 induces aberrant splicing, understanding of its consequences on the proteome and downstream cellular processes is limited $(9,10)$. We found that the $S F 3 B 1^{M U T}$ proteome has a dominant pattern of protein downregulation in genes affected by aberrant splicing. For many, the likely mechanism is NMD-induced mRNA downregulation. For others, it is likely translational or posttranslational, which might occur, for example, through impaired stability of proteins harboring indels created by cryptic splicing. We also found evidence that protein with a novel in-frame peptide insertion was expressed and enriched in $S F 3 B 1^{M U T}$ cells, in the form of cryptic SLC3A2. We suspect this was detectable because SLC3A2 is a highly abundant protein, but this finding does suggest that other, less abundant cryptic proteins may also be present in $S F 3 B 1^{M U T}$ cells. Whether - or how-these various protein alterations contribute to the oncogenicity of SF3B1 mutations is unknown. Tumor suppressor haploinsufficiency has been suggested as one possible mechanism (9), and there were tumor suppressor proteins downregulated by $S F 3 B 1^{M U T}$ in our study, such as NF1 and FANCD2 (Supplemental Table 7). An additional possibility is that cryptic proteins enriched in $S F 3 B 1^{M U T}$ cells could serve a gain-of-function oncogenic role, as has been suggested for the Notch pathway gene DVL2 in CLL (45). At the same time, such cryptic proteins might have implications for immune recognition of $S F 3 B 1^{M U T}$ cancers. While most cryptic transcripts induced by mutant $S F 3 B 1$ are also detectable-albeit at much lower levels-in $S F 3 B 1^{W T}$ cells, some could be sufficiently specific to $S F 3 B 1^{M U T}$ cells so as to produce cryptic peptides that would be recognized as nonself by the host immune system. If so, immunotherapeutic strategies aimed at targeting these putative missplicing-associated neo-antigens may afford therapeutic benefit, a testable hypothesis that we are currently investigating.

Our findings also demonstrate that $S F 3 B 1$ mutations cause a specific deregulation of metabolic genes and reprogramming of cell metabolism, characterized by decreases in mitochondrial complex III formation, mitochondrial respiration, and flux through the serine synthesis pathway. In breast cancer, $S F 3 B 1$ mutations rarely occur in triple-negative cases, and it is notable that this receptor subtype is highly dependent on the serine synthesis pathway, in some cases exhibiting amplification of $P H G D H(36,37)$. While inverse association does not necessarily equal mutual exclusivity, it could be that one reason acquisition of SF3B1 mutations is disfavored in triple-negative breast tumors is due to their serine synthesis pathway-suppressing effects. It is also worth noting that a defining feature of MDS driven by $S F 3 B 1^{M U T}$ is the ring sideroblast, which represents a pathologic deposit of iron in the matrix of structurally abnormal mitochondria that is accompanied by aberrant heme metabolism $(4,46)$. As suppression of $P H G D H$ has 
recently been shown to directly disturb mitochondrial homeostasis and heme synthesis in endothelial cells, it is possible that $P H G D H$ missplicing and downregulation could contribute to mitochondrial dysfunction and altered heme metabolism in SF3B1 ${ }^{\text {MUT }}$ MDS (32). Moreover, impairment of the electron transport chain can itself produce ring sideroblasts, as exemplified by the sideroblastic anemia caused by germline mutations in the ETC gene NDUFB11 (47). This raises the possibility that missplicing and downregulation of the ETC gene UQCC1 by SF3B1 ${ }^{M U T}$ could contribute to ring sideroblast formation in MDS. We believe future studies are warranted to investigate how the specific disease phenotypes of $S F 3 B 1^{M U T}$ cancers are influenced by aberrant splicing of metabolic genes.

It is also tempting to speculate that, in addition to shaping the metabolic behavior of $S F 3 B 1^{M U T}$ cells, reprogramming of energy metabolism could provide an advantage in the initiation and progression of $S F 3 B 1^{M U T}$ cancers. Indeed, metabolic reprogramming is a veritable hallmark of cancer that can drive tumorigenesis through rewiring of nutrient utilization and changes in metabolite-driven gene regulation (48). However, we believe it remains fundamentally mysterious how SF3B1 mutations confer a clonal advantage to cells, and that mystery was highlighted by our observations that knocking mutant SF3B1 into untransformed breast epithelial cells slowed rather than transformed their growth (Supplemental Figure 7). Whatever they are, the advantages of $S F 3 B 1$ mutations are likely to be contingent upon the genetic coconspirators and epigenetic differentiation of the cell, and it will be interesting to explore, in additional models, what environmental and cell-intrinsic conditions unleash the oncogenic properties of SF3B1 mutations, and whether metabolic reprogramming is involved.

Whether cause or consequence of cancer, the altered metabolic properties we observed in $S F 3 B 1^{M U T}$ cells offered a novel vulnerability to deprivation of the nonessential amino acid serine. Previous studies have shown anticancer activity for serine restriction, but less is known about the molecular determinants of cancer responsiveness to this treatment $(24,31,43,44,49)$. Maddocks et al. showed that p53 mutation sensitizes HCT116 colon cancer cells to serine deprivation by favoring the diversion of residual serine into nucleotide rather than glutathione synthesis, but this group also found that a murine p53-mutant pancreas cancer model was resistant to serine deprivation, demonstrating the context-dependence of p53 mutation on this phenotype $(43,44)$. We observed heightened sensitivity to serine starvation associated with SF3B1 mutation in multiple contexts, including 2 independent breast epithelial cell knockin models, a breast cancer knockin model, a leukemia knockin model, and naturally mutated leukemia cells. We also found evidence that the mechanism underlying this vulnerability involves missplicing and downregulation of the serine synthesis pathway enzyme $P H G D H$, as restored expression of this enzyme was able to rescue growth without SG in $S F 3 B 1^{K 700 E}$ breast epithelial cell knockins and $S F 3 B 1^{M U T}$ leukemia cell knockins. Given the diversity of genes affected by $S F 3 B 1^{M U T}$ missplicing, it would not be surprising if altered activity of other metabolic proteins in addition to $P H G D H$ contributes to this phenotype. Nonetheless, we describe here a novel form of synthetic lethality for $S F 3 B 1^{M U T}$ cells, in which a predictable missplicing event is tolerated by itself but not in the context of additional metabolic stress. The proposed therapy also has a target-exogenous serine-that is nonessential to normal cells, as serine starvation appears well tolerated in mice $(31,43)$. This favorable toxicity profile may, in turn, allow dietary serine restriction to be combined with other treatments, as has been done in mice with biguanides and glutaminase inhibitors $(24,44,50)$. It is also conceivable that in vivo administration of a serine-depleting enzyme could produce even greater serine deprivation and anticancer activity while still remaining tolerable to the host. Indeed, asparaginase is a backbone of curative therapy for acute lymphoblastic leukemia, and arginine-depleting enzymes have shown signs of anticancer activity in patients (51). Whether serine deprivation will be tolerable or effective in humans is unknown, but our findings provide a rationale for investigating this therapeutic strategy in patients with SF3B1-mutant cancers.

\section{Methods}

Cell lines. HEK293T cells were from ATCC and grown in DMEM/10\% FBS. MCF-10A cells were from ATCC and grown in DMEM/F12 with $5 \%$ horse serum, $20 \mathrm{ng} / \mathrm{mL}$ EGF, $10 \mu \mathrm{g} / \mathrm{mL}$ insulin, $0.5 \mu \mathrm{g} / \mathrm{mL}$ hydrocortisone, and $0.1 \mu \mathrm{g} / \mathrm{mL}$ cholera toxin. Gene-targeted MCF-10A derivatives were grown in the same media, except PIK3CA ${ }^{\mathrm{E} 545 \mathrm{~K}}$ knockins (used as a control for the experiment shown in Supplemental Figure 7), which were cultured without EGF. hTERT-IMEC cells were a gift from JW Shay (UT Southwestern Medical Center, Dallas, TX) and were cultured in MCF-10A media, except horse serum was substituted with 2\% charcoal dextran stripped FBS (52). HNT34, MUTZ, and CMLT1 cells were from DSMZ. Nalm6 WT and $S F 3 B 1^{+/ H 662 Q}$ cells were from Horizon discovery, as were T47D WT, $S F 3 B 1^{+/ K 700 E}$, and $S F 3 B 1^{+/ K 700 K}$ clones. K562, MOLM13, MONOMAC6, MV411, TF1, and KASUMI1 cells were gifts from LP Gondek (Johns Hopkins University, Baltimore, MD). KG1 cells were a gift from RA Casero (Johns Hopkins University, Baltimore, MD). All leukemia cells were grown in RPMI/20\% FBS, with supplements as follows: $10 \mathrm{ng} / \mathrm{mL}$ GM-CSF for MUTZ3, $2 \mathrm{ng} / \mathrm{mL}$ GM-CSF for TF1, and $10 \mu \mathrm{g} / \mathrm{mL}$ insulin for MONOMAC6. T47D cells were grown in RPMI/10\% FBS. SF3B1 genotype was confirmed in all lines by Sanger sequencing of exons 13-24. STR cell line authentication and mycoplasma testing was done upon receipt and routinely thereafter, with last testing done April 2018.

Gene targeting. Gene targeting was carried out using recombinant AAV technology as previously described (53). Briefly, AAV targeting vectors were produced by ligating PCR-generated homology arms spanning intron 12 to intron 17 of the SF3B1 gene into a pSEPT-containing AAV plasmid backbone (Agilent), which confers geneticin resistance through a promoterless IRES-NEO cassette. Site-directed mutagenesis was then performed to generate the K700E and R702R alterations (Supplemental Figure 1). Virus was prepared by transfecting HEK-293T cells with pHelper, pRC (Agilent) and respective SF3B1 AAV plasmids. Breast epithelial cells were infected, and single geneticin-resistant clones were isolated and assayed for integration of the targeting cassette with PCR. Successfully targeted clones were exposed to Cre-expressing adenovirus to remove the neomycin cassette. All knockins underwent Sanger sequencing of genomic DNA and cDNA to ensure presence and transcription of the intended mutation. Primer sequences used for homology arm amplification are shown in Supplemental Table 10.

Transcriptome analysis. Total RNA was harvested from cultured cells using RNeasy and Qiashredder kits (Qiagen). RNA-seq libraries were constructed using the Illumina TruSeq RNA Sample Preparation 
Kit v3. Sequencing was performed on an Illumina HiScanSQ system to obtain a total of more than 40 million paired-end reads of $101 \mathrm{bp}$ in length per replicate. Reads were aligned using TopHat (version 2.0.10) against the GRCh38/hg38 version of the human genome, and assembly was carried out with Cufflinks pipeline. Differential mRNA expression between mutant and control cells was analyzed with DESeq2 from read counts obtained using HTSeq (htseq-count) and GENCODE (release 26) gene annotations. Four mutant MCF10A replicates ( 2 from the K700E1 clone and 2 from K700E2) and 4 WT replicates ( 2 from R702R1 and 2 from TWT1) were compared. Splice junctions were identified by RSeQC (version 2.6.4) and quantified using DEXseq, and PSI values for junctions were determined by dividing the read counts for a given junction by all read counts that included either the donor or acceptor site. Junctions with coverage of at least 10 reads in mutant samples were included, and a PSI threshold of 5 or more was employed. Novel junctions were those not found in major transcriptome databases, including KnownGene, AceView, and Ensembl. Splicing events were classified using exon-intron annotations from the GRCh38/hg38 reference assembly. Because it was shown to comprise a very minor fraction of $S F 3 B 1^{M U T}$-associated missplicing events (9), intron retention was not included in the analysis. A junction was classified as NMD-sensitive if it produced a premature termination codon (PTC) more than 55 nucleotides upstream of the last exon-exon boundary (54). Transcriptome data, including raw fastq files, identified junctions, and assembled transcript annotations, were deposited in NCBI's Gene Expression Omnibus (GEO) and are accessible through GEO Series accession number GSE134475 (http://www.ncbi. nlm.nih.gov/geo/query/acc.cgi?acc=GSE134475). For splice junction usage between TCGA breast tumors and isogenic MCF-10A cells, all splicing events identified as upregulated in $S F 3 B 1^{M U T}$ breast tumors by Darman et al. were included except intron retention. For wholegene mRNA expression comparison between isogenic MCF-10As and TCGA/METABRIC data sets, the latter was obtained through cBioportal and those genes with altered expression in $S F 3 B 1^{M U T}$ vs $S F 3 B 1^{W T}$ tumors at a false discovery $q$ value of less than 0.05 were included. The criteria for including SF3B1 mutations from the TCGA, METABRIC, and Beat AML data sets in the $S F 3 B 1^{M U T}$ group were that the mutations are recurrent in primary human cancers, create in-frame amino acid changes, and are located in the HEAT repeats domain, as DeBoever et al. previously showed that mutations outside the HEAT repeats do not exhibit the same cryptic splicing pattern (55). Validation of aberrant junctions was done with endpoint PCR on cDNA from cells using primers designed to amplify both the cryptic junction of interest and its paired canonical junction. These primers sequences are provided in Supplemental Table 10.

Proteome analysis. SILAC-based mass spectrometry of MCF-10A cells was performed as previously described (19). Briefly, 2 independent K700E clones and a TWT clone were propagated for more than 7 population doublings in DMEM/F12 SILAC media deficient in both L-lysine and L-arginine (Thermo Fisher Scientific) and supplemented with light lysine (K) and arginine (R) for light, ${ }^{2} \mathrm{H} 4-\mathrm{K}$ and ${ }^{13} \mathrm{C} 6-\mathrm{R}$ for medium state, and ${ }^{13} \mathrm{C}^{1} 6{ }^{5} \mathrm{~N} 2-\mathrm{K}$ and ${ }^{13} \mathrm{C}^{1} 6{ }^{5} \mathrm{~N} 4-\mathrm{R}$ for heavy state labeling (Cambridge Isotope Laboratories). Cells were lysed in 9M urea in ammonium bicarbonate buffer and equal amounts of proteome from the 3 SILAC-labeled states were mixed. Proteome was subjected to reduction and alkylation with $10 \mathrm{mM}$ DTT and $20 \mathrm{mM}$ iodoacetamide, respectively. Sequencing grade trypsin (Promega) was added in a 1:20 ratio followed by overnight trypsin digestion at $37^{\circ} \mathrm{C}$. Peptides were prefractionated by basic RPLC chromatography using Waters X-bridge C18 column, and 12 fractions were collected. Each fraction was subjected to LC-MS/MS analysis on a Thermo Orbitrap Fusion Lumos mass spectrometer. Peptides were separated on an EASY-Spray analytical column ( $2 \mu \mathrm{m}, 50 \mathrm{~cm}$, Thermo Scientific) over a 180-minute gradient. The data were acquired in a data-dependent manner in top speed mode over 3 seconds. Peptides were fragmented in higher-energy collisional dissociation (HCD) mode at $35 \%$ collision energy. Dynamic exclusion of 30 seconds was included in the method. Data were analyzed in 2 ways. First, for routing, protein quantitation spectral data were searched against Human RefSeq data set (release 73) using SILAC-based quantitation method. Second, for identification of novel splice junction peptides, the spectral data were searched against a 3 frame translated transcriptomic data obtained as a part of the same study. Peptide identification and quantification was done using Proteome Discoverer suite (version 2.1, Thermo Fisher Scientific). Peptide spectral matches qualifying $1 \%$ FDR by decoy database search method were accepted. Western blotting was performed as previously described (56). The following antibodies were used: anti-UQCC1 (Sigma-Aldrich, HPA034875), anti-UQCC2 (Sigma-Aldrich, HPA039111), anti-UQCRFS1 (Abcam, ab14746), antiUQCRC1 (Abcam, ab110252), anti-UQCRQ (Abcam, ab110255), antiMUT (Abcam, ab133672), anti-DLST (Abcam, ab187699), anti-PHGDH (Sigma-Aldrich, HPA021241), anti- $\beta$-Actin (Cell Signaling, 4970S), anti-GAPDH (Cell Signaling, 5174).

Gene ontology analysis. Proteins identified as downregulated or upregulated at $\log _{2}$ abundance ratios of \pm 0.5 in $S F 3 B 1^{K 700 E}$ MCF-10A cells by SILAC were submitted to GO biological process enrichment analysis through the Gene Ontology Consortium (http://geneontology.org) (57).

Overexpression experiments. For UQCC1 overexpression, canonical UQCC1 was PCR amplified from cDNA made from MCF-10A cells and cloned into the lentiviral vector pLX301 (Addgene plasmid 25895). The pEXP7-TET cassette from the BP Clonase Enzyme Kit (Thermo Fisher Scientific) was cloned into pLX301 as a vector control. Lentivirus was produced by transfection of 293T cells with pMD2.6 (Addgene plasmid 12259), psPAX2 (Addgene plasmid 12260), and the pLX301 derivatives. For PHGDH overexpression in MCF-10A and hTERT-IMEC cells, we made lentivirus as above, but with pLJM5-WT PHGDH lentiviral vector (Addgene plasmid 83901). For GFP control virus, we replaced the PHGDH ORF in pLJM5-WT PHGDH with GFP from pmaxGFP (Lonza) and produced lentivirus otherwise as above. Breast epithelial cells were then infected with lentivirus, selected with puromycin (UQCC1) or hygromycin $(P H G D H)$, and collected as independent polyclonal pools of stably expressing cells. For PHGDH overexpression in Nalm6 cells, the full-length $P H G D H$ open reading frame was PCR amplified from cDNA from MCF-10A cells, inserted into the lentiviral vector pLX301, and lentivirus produced as above. The pEXP7-TET-pLX301 virus was used as a vector control, as above. Independent polyclonal Nalm6 pools of transduced cells were selected with puromycin.

Metabolite quantification. For MCF-10As, gene targeting control cells (R702R1) were grown over 4 days with or without $5 \mathrm{mM}$ metformin, and $S F 3 B 1^{K 7 O O E}$ knockin cells were also grown for 4 days, but seeded at a higher starting density so that cell confluency was similar by the end of the experiment. Media was changed at 48 and 72 hours. At the time of harvest, cell counting was done on extra replicates of cells cultured in the same conditions and used just for the purpose of determining the volume of trypsinized cells needed to pellet $5 \times 10^{6}$ 
cells in the samples of interest, to minimize processing time. Cells were trypsinized for approximately 1 minute with $0.25 \%$ trypsin, and the predetermined volumes were spun and pellets snap-frozen in liquid nitrogen. The protein content of samples was then quantified using the remaining trypsinized cells with Pierce 660 per the manufacturer's protocol for use in normalization of final metabolite levels. The frozen pellets were shipped to Biocrates for extraction and quantification of energy metabolites by mass spectrometry. Orthogonal validation of aspartate levels was performed using similar culture conditions, but with independent isogenic MCF-10A clones using the Aspartate Assay Kit from Sigma-Aldrich (MAK095) per the manufacturer's protocol.

For hTERT-IMECs, WT and $S F 3 B 1^{K 700 E}$ knockin cells were grown in 10-cm dishes in +SG and -SG media for 42 hours, and media was refreshed, but with ${ }^{13} \mathrm{C}$-glucose for an additional 6 hours. Media was then withdrawn from the dishes and ice-cold $80 \% \mathrm{MeOH}$ was added directly to cells. Cells were scraped off the dish and flash frozen in liquid nitrogen. Samples were vortexed and stored at $-80^{\circ} \mathrm{C}$ for at least 2 hours to precipitate protein. Protein obtained from cells during metabolite extraction was quantified using BCA per the manufacturer's protocol (MilliporeSigma) and protein levels were used for normalization of total ion abundance levels in the samples. Metabolite-containing supernatant was isolated after centrifugation at $15,000 \mathrm{~g}$ for $10 \mathrm{~min}$ utes and dried under nitrogen gas and frozen at $-80^{\circ} \mathrm{C}$ for subsequent analysis by liquid-chromatography mass spectrometry (LC-MS). Dried metabolite extracts were resuspended in $50 \%$ acetonitrile solution. LC-MS-based metabolomics profiling was performed on an Agilent LC-MS system consisting of an Agilent 1290 Infinity Binary UHPLC pump and a 6230 time-of-flight mass spectrometer. Chromatographic separations were performed using an Agilent 1290 ultra high-performance liquid chromatography system with a wellplate autosampler. An ion pairing method was developed using a C18 column (Agilent Zorbax Extend C18, $2.1 \times 150$ mm, $1.8 \mu \mathrm{m}$ ) with tributylamine as an ion-pairing agent, which enables the detection of most of the metabolites in central carbon metabolism. The LC parameters were as follows: autosampler temperature, $4^{\circ} \mathrm{C}$; injection volume, $2 \mu \mathrm{L}$; column temperature, $40^{\circ} \mathrm{C}$; and flow rate, $0.25 \mathrm{~mL} / \mathrm{min}$. The solvents and optimized gradient conditions for LC were as follows: Solvent A, 97\% water/3\% methanol containing $5 \mathrm{mM}$ TBA and $5.5 \mathrm{mM}$ acetic acid; Solvent B, methanol containing $5 \mathrm{mM}$ TBA and $5.5 \mathrm{mM}$ acetic acid; a nonlinear gradient from 0\% B to $99 \%$ B in 22 minutes with 5 minutes of post-run time. A 6520 accurate-mass Q-TOF LC-MS system (Agilent) equipped with a dual electrospray (ESI) ion source was operated in negative-ion mode for metabolic profiling. The optimized ESI Q-TOF parameters for MS experiments were: ion polarity, negative; gas temperature, $325^{\circ} \mathrm{C}$; drying gas, $10 \mathrm{~L} / \mathrm{min}$; nebulizer pressure, 45 psig; capillary voltage, $4000 \mathrm{~V}$; fragmentor, $140 \mathrm{~V}$; skimmer, $65 \mathrm{~V}$; mass range, $50-1100 \mathrm{~m} / \mathrm{z}$; acquisition rate, 1.5 spectra/s; instrument state, extended dynamic range $(1700 \mathrm{~m} / \mathrm{z}, 2 \mathrm{GHz})$. Spectra were internally mass calibrated in real time by continuous infusion of a reference mass solution using an isocratic pump connected to a dual sprayer feeding into an electrospray ionization source. Data were acquired with MassHunter Acquisition software (Agilent Technologies).

For quantification of oxygen consumption rate, isogenic MCF-10A cells were seeded at 28,000 cells per well in Seahorse XF96 Analyzer plates in regular media and incubated at $37^{\circ} \mathrm{C}$ with $5 \% \mathrm{CO}_{2}$ overnight. Media was then removed, cells were washed, Seahorse media (RPMI $+17.5 \mathrm{~mm}$ glucose $+1 \mathrm{mM}$ pyruvate $+2 \mathrm{mM}$ glutamine) was added to wells, and the plate was incubated at $37^{\circ} \mathrm{C}$ without $\mathrm{CO}_{2}$ for 1 hour. The plate was then loaded into the Seahorse machine and readings of oxygen and $\mathrm{pH}$ levels were taken before and after injections of $3 \mu \mathrm{M}$ oligomycin, $4 \mu \mathrm{M}$ FCCP, $2 \mu \mathrm{M}$ antimycin, and $2 \mu \mathrm{M}$ rotenone. Oxygen consumption rate and extracellular acidification rate were analyzed using Wave 2.4 software.

Serine and glycine starvation. Amino acid-free RPMI powder (US Biological, R9010-01) was individually reconstituted with RPMI-standard concentrations of amino acids (Sigma-Aldrich) as needed for experimental conditions. All serum used for starvation experiments was dialyzed FBS (dFBS, Thermo Fisher Scientific, 26400044). For MCF-10A and hTERT-IMEC cells, which were found capable of growing in RPMI, supplements for breast epithelial cell growth (EGF, insulin, cholera toxin, hydrocortisone) were added to reconstituted RPMI, with the same concentrations used for DMEM/F12 as detailed above, except $5 \%$ horse serum was substituted with 5\% dFBS. MCF-10A isogenic cells were grown in +SG or -SG media for 9 days with media changes every 2 days, and viable cells were then counted with a Vi-Cell $\mathrm{XR}$ cell counter. Isogenic hTERT-IMEC cells were grown in $+\mathrm{SG}$ and -SG media for 6 days, at which point viable cells were counted. MCF10A and hTERT-IMEC cells overexpressing either GFP or PHGDH were cultured in $+S G$ or -SG in the same manner, as were phosphohydroxypyruvate (PHP) rescue experiments, but with increasing concentrations (0-1 mM) of PHP. For Nalm6 isogenic cells, RPMI + 20\% dFBS with or without SG was used, cells were seeded at a starting density of $10^{6}$ cells $/ \mathrm{mL}$, equal volumes of additional +SG or -SG media were added at 48 hours and 96 hours, and cell mass/viability was determined on day 6 with alamarBlue, per the manufacturer's protocol. Other leukemia cell lines were cultured in +SG or -SG media using RPMI $+20 \%$ dialyzed FBS and, where required, the same supplements (GM-CSF, insulin) as in their routine culture media. Cells were seeded at a starting density of $10^{6}$ cells $/ \mathrm{mL}$, an equal volume of additional + SG or -SG media was added at 48 hours, and cell mass/viability was quantified by alamarBlue on day 4 . For starvation of serine-vs.-glycine-vs.-both experiments, HNT34 and MUTZ3 cells were seeded in respective media at $10^{6}$ cells $/ \mathrm{mL}$ and viable cells counted at 48 hours. For gradient starvation of serine, HNT34 cells were seeded at $10^{6}$ cells $/ \mathrm{mL}$ in media containing increasing increments of serine, from 0 to the standard RPMI concentration of $285 \mu \mathrm{M}$, and viable cells were counted at 96 hours. For isogenic T47Ds, cells were seeded into 96-well low attachment plates (Corning) at a density of 5000 cells per well in $200 \mu \mathrm{L}$ of $\mathrm{RPMI} / 10 \% \mathrm{dFBS}$ with or without SG. Plates were spun at $1000 \mathrm{~g}$ in a bench top centrifuge to form 3D spheroids and media topped up to 300 $\mu \mathrm{L}$ per well the next day. Spheroids were grown for 16 days and quantified by cell titre glo viability assays (Promega). To replenish media, 100 $\mu \mathrm{L}$ was removed and replaced with fresh media.

Mouse xenograft studies. Nine-week-old female NOD-scid IL2Rg ${ }^{\text {null }}$ (NSG) and 5-week old male NOD-scid IL2Rgull-3/GM/SF (NSGS) mice were obtained from The Jackson Laboratory. Mice were started on -SG or + SG pellets (Test Diet 5CC7) beginning 1 week before cell injections. For HNT34 cells, $10^{7}$ cells were injected into the flanks of NSG mice on day 0. Mice were continued on +SG or -SG diets, and tumors in the $+\mathrm{SG}$ group first became measurable at week 5 . Tumor measurements were made weekly for 4 weeks, after which the mice were euthanized. For MUTZ3 cells, $10^{7}$ cells were injected into the flanks of NSGS mice on day 0, and mice were euthanized at week 4 , at which point tumors were dissected from flanks and measured. 
Proliferation and 3D growth. Baseline proliferation in MCF-10A and hTERT-IMEC cells was determined in standard DMEM/F12-supplemented media. Media was changed every 2 days, and cells were trypsinized and counted on day 6 (MCF-10A) or day 5 (hTERT-IMECs). For growth factor-independent proliferation, MCF-10A cells were seeded at low density in T25 flasks and grown in standard DMEM/F12-supplemented media, but without EGF for 3 weeks, at which point they were stained with crystal violet and photographed. For mammosphere formation, isogenic MCF-10A cells were grown in standard media combined with Matrigel for 10 days, at which point representative photographs were taken. Soft agar colony formation assays were performed as previously described (56).

Statistics. Significance of $\triangle$ PSI of splice junctions between mutant and control MCF-10A cells was tested using a moderated, 2-tailed $t$ test with $P$ less than 0.01 and Benjamini-Hochberg correction $q$ less than 0.05 . Genes differentially regulated in $S F 3 B 1^{M U T}$ breast tumors from TCGA and METABRIC were included at FDRs of less than 0.10 and less than 0.05 , respectively, due to the larger overall number of tumors in the METABRIC cohort. For gene ontology analysis, processes enriched at $P$ less than 0.05 by Fisher's exact test with $q$ less than 0.05 by Benjamini-Hochberg FDR correction were first captured and then further filtered for those with a fold enrichment of greater than 3. For xenotransplantation, tumor volumes and statistical comparisons were calculated as previously described (58). For growth rescue with PHP, Dunnett's test was applied to compare control cells with multiple increasing doses of PHP. For all other experiments, a 2-tailed Student's $t$ test with $P$ less than 0.05 was used to test for significance between groups. All graphs show mean \pm SEM.

Study approval. The animal care and use protocol of this study was approved by the IACUC of the Johns Hopkins University.

\section{Author contributions}

WBD, EH, LPG, DSK, RN, ESC, SD, RDL, A Pandey, J Lauring, and BHP designed research. WBD, EH, NW, LPG, DSK, AR, ESC, BR, LZ, RDL, DS, TG, DJZ, AM, J Lee, AJC, MR, MT, AA, JD, KC, DC, and RMM conducted experiments. WBD, EH, NW, LPG, DSK, AR, RN, ESC, BR, SD, LZ, RDL, DS, TG, AKM, A Pandey, DJZ, AM, J Lee, AJC, JD, AED, KC, DC, RMM, A Patil, PJH, J Lauring, and BHP analyzed data. WBD, ESC, DSK, AKM, A Patil, JD, AED, and BHP wrote the manuscript.

\section{Acknowledgments}

This work was supported by grants from the Conquer Cancer Foundation, Breast Cancer Research Foundation, Breast Cancer Now, Ladies Auxillary of the Veterans of Foreign Wars, Lilly Innovation Fellowship Award, and Department of Defense (W81XWH-17-1-0035) (to WBD); NIH K08HL136894 (to LPG); and the Susan G. Komen Foundation and Abbvie (to BP).

Address correspondence to: W. Brian Dalton, The Sidney Kimmel Comprehensive Cancer Center at Johns Hopkins, 1650 Orleans Street, CRBI Room 285, Baltimore, Maryland 21231, USA. Phone: 410.614.6173; Email: wdalton2@jhmi.edu.
1. Yoshida K, et al. Frequent pathway mutations of splicing machinery in myelodysplasia. Nature. 2011;478(7367):64-69.

2. Campbell JD, et al. Distinct patterns of somatic genome alterations in lung adenocarcinomas and squamous cell carcinomas. Nat Genet. 2016;48(6):607-616.

3. Armenia J, et al. The long tail of oncogenic drivers in prostate cancer. Nat Genet. 2018;50(5):645-651.

4. Papaemmanuil E, et al. Somatic SF3B1 mutation in myelodysplasia with ring sideroblasts. $N \mathrm{EnglJ}$ Med. 2011;365(15):1384-1395.

5. Wang L, et al. SF3B1 and other novel cancer genes in chronic lymphocytic leukemia. $N$ Engl J Med. 2011;365(26):2497-2506.

6. Harbour JW, Roberson ED, Anbunathan H, Onken MD, Worley LA, Bowcock AM. Recurrent mutations at codon 625 of the splicing factor $S F 3 B 1$ in uveal melanoma. Nat Genet. 2013;45(2):133-135.

7. Biankin AV, et al. Pancreatic cancer genomes reveal aberrations in axon guidance pathway genes. Nature. 2012;491(7424):399-405.

8. Stephens PJ, et al. The landscape of cancer genes and mutational processes in breast cancer. Nature. 2012;486(7403):400-404.

9. Darman RB, et al. Cancer-associated SF3B1 hotspot mutations induce cryptic 3' splice site selection through use of a different branch point. Cell Rep. 2015;13(5):1033-1045.

10. Alsafadi S, et al. Cancer-associated SF3B1 mutations affect alternative splicing by promoting alternative branchpoint usage. Nat Commun. 2016;7:10615.

11. Zhou Q, et al. A chemical genetics approach for the functional assessment of novel cancer genes. Cancer Res. 2015;75(10):1949-1958.

12. Hsu J, et al. Reprogramming identifies functionally distinct stages of clonal evolution in myelodysplastic syndromes. Blood. 2019;134(2):186-198.

13. Maguire SL, et al. SF3B1 mutations constitute a novel therapeutic target in breast cancer. JPathol. 2015;235(4):571-580.

14. Fu X, et al. SF3B1 mutation is a poor prognostic indicator in luminal $\mathrm{B}$ and progesterone receptor-negative breast cancer patients. Oncotarget. 2017;8(70):115018-115027.

15. Christenson ES, et al. Single-nucleotide polymorphism leading to false allelic fraction by droplet digital PCR. Clin Chem. 2017;63(8):1370-1376.

16. Cancer Genome Atlas Network. Comprehensive molecular portraits of human breast tumours. Nature. 2012;490(7418):61-70.

17. Pereira B, et al. The somatic mutation profiles of 2,433 breast cancers refines their genomic and transcriptomic landscapes. Nat Commun. 2016;7:11479.

18. Tress ML, Abascal F, Valencia A. Alternative splicing may not be the key to proteome complexity. Trends Biochem Sci. 2017;42(2):98-110.

19. Wu X, et al. Activation of diverse signalling pathways by oncogenic PIK3CA mutations. Nat Commun. 2014;5:4961.

20. Tucker EJ, et al. Mutations in the UQCC1interacting protein, UQCC2, cause human complex III deficiency associated with perturbed cytochrome b protein expression. PLoS Genet. 2013;9(12):e1004034.

21. Wanschers BF, et al. A mutation in the human
CBP4 ortholog UQCC3 impairs complex III assembly, activity and cytochrome b stability. Hum Mol Genet. 2014;23(23):6356-6365.

22. Birsoy K, Wang T, Chen WW, Freinkman E, Abu-Remaileh M, Sabatini DM. An essential role of the mitochondrial electron transport chain in cell proliferation is to enable aspartate synthesis Cell. 2015;162(3):540-551.

23. Sullivan LB, Gui DY, Hosios AM, Bush LN, Freinkman E, Vander Heiden MG. Supporting aspartate biosynthesis is an essential function of respiration in proliferating cells. Cell. 2015;162(3):552-563.

24. Gravel SP, et al. Serine deprivation enhances antineoplastic activity of biguanides. Cancer Res. 2014;74(24):7521-7533.

25. Lee HJ, et al. Proteomic and metabolomic characterization of a mammalian cellular transition from quiescence to proliferation. Cell Rep. 2017;20(3):721-736.

26. Sullivan LB, et al. Aspartate is an endogenous metabolic limitation for tumour growth. Nat Cell Biol. 2018;20(7):782-788.

27. Garcia-Bermudez J, et al. Aspartate is a limiting metabolite for cancer cell proliferation under hypoxia and in tumours. Nat Cell Biol. 2018;20(7):775-781.

28. Cardaci S, et al. Pyruvate carboxylation enables growth of SDH-deficient cells by supporting aspartate biosynthesis. Nat Cell Biol. 2015;17(10):1317-1326.

29. Pollari S, et al. Enhanced serine production by bone metastatic breast cancer cells stimulates osteoclastogenesis. Breast Cancer Res Treat. 
2011;125(2):421-430.

30. Ou Y, Wang SJ, Jiang L, Zheng B, Gu W. p53 Protein-mediated regulation of phosphoglycerate dehydrogenase (PHGDH) is crucial for the apoptotic response upon serine starvation. J Biol Chem. 2015;290(1):457-466.

31. Sullivan MR, et al. Increased serine synthesis provides an advantage for tumors arising in tissues where serine levels are limiting. Cell Metab. 2019;29(6):1410-1421.e4.

32. Vandekeere S, et al. Serine synthesis via $P H G D H$ is essential for heme production in endothelial cells. Cell Metab. 2018;28(4):573-587.e13.

33. Possemato R, et al. Functional genomics reveal that the serine synthesis pathway is essential in breast cancer. Nature. 2011;476(7360):346-350.

34. Pacold ME, et al. A PHGDH inhibitor reveals coordination of serine synthesis and one-carbon unit fate. Nat Chem Biol. 2016;12(6):452-458.

35. Locasale JW, et al. Phosphoglycerate dehydrogenase diverts glycolytic flux and contributes to oncogenesis. Nat Genet. 2011;43(9):869-874.

36. Murphy JP, et al. The $\mathrm{NAD}^{+}$salvage pathway supports $P H G D H$-driven serine biosynthesis. Cell Rep. 2018;24(9):2381-2391.e5.

37. Lau CE, Tredwell GD, Ellis JK, Lam EW, Keun HC. Metabolomic characterisation of the effects of oncogenic PIK3CA transformation in a breast epithelial cell line. Sci Rep. 2017;7:46079.

38. Gao X, et al. Serine availability influences mitochondrial dynamics and function through lipid metabolism. Cell Rep. 2018;22(13):3507-3520.

39. Chaneton B, et al. Serine is a natural ligand and allosteric activator of pyruvate kinase M2. Nature. 2012;491(7424):458-462.

40. de Olza MO, Oliva M, Hierro C, Matos I, Martin-
Liberal J, Garralda E. Early-drug development in the era of immuno-oncology: are we ready to face the challenges? Ann Oncol. 2019;29(8):1727-1740.

41. Dolatshad H, et al. Disruption of $S F 3 B 1$ results in deregulated expression and splicing of key genes and pathways in myelodysplastic syndrome hematopoietic stem and progenitor cells. Leukemia. 2015;29(5):1092-1103.

42. Tyner JW, et al. Functional genomic landscape of acute myeloid leukaemia. Nature. 2018;562(7728):526-531.

43. Maddocks OD, et al. Serine starvation induces stress and p53-dependent metabolic remodelling in cancer cells. Nature. 2013;493(7433):542-546.

44. Maddocks ODK, et al. Modulating the therapeutic response of tumours to dietary serine and glycine starvation. Nature. 2017;544(7650):372-376

45. Wang $\mathrm{L}$, et al. Transcriptomic characterization of SF3B1 mutation reveals its pleiotropic effects in chronic lymphocytic leukemia. Cancer Cell. 2016;30(5):750-763.

46. Yang Z, et al. Delayed globin synthesis leads to excess heme and the macrocytic anemia of Diamond Blackfan anemia and $\operatorname{del}(5 \mathrm{q})$ myelodysplastic syndrome. Sci Transl Med. 2016;8(338):338ra67.

47. Lichtenstein DA, et al. A recurring mutation in the respiratory complex 1 protein NDUFB11 is responsible for a novel form of $\mathrm{X}$-linked sideroblastic anemia. Blood. 2016;128(15):1913-1917.

48. Pavlova NN, Thompson CB. The emerging hallmarks of cancer metabolism. Cell Metab. 2016;23(1):27-47.

49. Polet F, et al. Reducing the serine availability complements the inhibition of the glutamine metabolism to block leukemia cell growth. Onco- target. 2016;7(2):1765-1776.

50. Matre $P$, et al. Inhibiting glutaminase in acute myeloid leukemia: metabolic dependency of selected AML subtypes. Oncotarget. 2016;7(48):79722-79735.

51. Szlosarek PW, et al. Arginine deprivation with pegylated arginine deiminase in patients with argininosuccinate synthetase 1-deficient malignant pleural mesothelioma: a randomized clinical trial. JAMA Oncol. 2017;3(1):58-66.

52. Herbert BS, Wright WE, Shay JW. p16(INK4a) inactivation is not required to immortalize human mammary epithelial cells. Oncogene. 2002;21(51):7897-7900.

53. Gustin JP, et al. Knockin of mutant PIK3CA activates multiple oncogenic pathways. Proc Natl Acad Sci U S A. 2009;106(8):2835-2840.

54. Rivas MA, et al. Human genomics. Effect of predicted protein-truncating genetic variants on the human transcriptome. Science. 2015;348(6235):666-669.

55. DeBoever C, et al. Transcriptome sequencing reveals potential mechanism of cryptic 3' splice site selection in SF3B1-mutated cancers. PLoS Comput Biol. 2015;11(3):e1004105.

56. Zabransky DJ, et al. HER2 missense mutations have distinct effects on oncogenic signaling and migration. Proc Natl Acad Sci U S A. 2015;112(45):E6205-E6214.

57. The Gene Ontology Consortium. Expansion of the Gene Ontology knowledgebase and resources. Nucleic Acids Res. 2017;45(D1):D331-D338.

58. Wang GM, et al. Single copies of mutant KRAS and mutant PIK3CA cooperate in immortalized human epithelial cells to induce tumor formation. Cancer Res. 2013;73(11):3248-3261. 